\title{
NRCL - A Model Building Approach to the Bernays-Schönfinkel Fragment (Full Paper)
}

\author{
Gábor Alagi ${ }^{1,2}$ and Christoph Weidenbach ${ }^{2}$ \\ ${ }^{1}$ Saarland University, Saarbrücken, Germany \\ ${ }^{2}$ Max-Planck-Institut für Informatik, Saarbrücken, Germany \\ \{galagi, weidenbach\}@mpi-inf.mpg.de
}

\begin{abstract}
We combine key ideas from first-order superposition and propositional CDCL to create the new calculus NRCL deciding the Bernays-Schönfinkel fragment. It inherits the abstract redundancy criterion and the monotone model operator from superposition. CDCL adds to NRCL the dynamic, conflict-driven search for an atom ordering inducing a model. As a result, in NRCL a false clause can be effectively found modulo the current model assumption. It guides the derivation of a first-order ordered resolvent that is never redundant. Similar to 1UIP-learning in CDCL, the learned resolvent induces backtracking and via propagation blocks the previous conflict state for the rest of the search. Since learned clauses are never redundant, only finitely many can be generated by NRCL on the BernaysSchönfinkel fragment, which provides a nice argument for termination.
\end{abstract}

\section{Introduction}

The Bernays-Schönfinkel fragment, also called Effectively Propositional fragment, or BS (or EPR) for short, is a meaningful and important fragment of classic first-order logic, where only constants are allowed as function symbols in the clause normal form.

This decidable and NEXPTIME-complete fragment has many applications, and over the years a number of calculi attempted to provide an efficient solution for BS problems. These approaches range from the early SEM and Mace systems 31] to the recent state-of-the-art solvers like iProver [14] and Darwin [7, but even general purpose first-order theorem provers provide specialized techniques for BS problems, like generalisation in Vampire [25, or specialized splitting techniques for SPASS introduced in [16] and [13.

In this paper, we give a brief introduction to our on-going research on solving BS problems with iterative model building. Our approach aims to work on fullfledged first-order candidate models instead of approximations, uses constrained literals for model representation, and learns new clauses to guide the search.

Our calculus, called NRCL or Non-Redundant Clause Learning, shares many principles with propositional SAT solving and superposition. For a detailed 
introduction to conflict-driven clause learning ( $C D C L)$, see the early article [29], or the more recent handbook 8]. The interested reader can get a thorough overview of superposition in the articles $3,4,32,23$.

The most closely related existing system is $\operatorname{DPLL}(S X)$ [27, a calculus aiming to lift the propositional CDCL algorithm through representing sets of clause ground instances with substitution sets.

Compared to DPLL(SX), our formulation depends less on the concrete constraint language, our choice of dismatching constraints provides more compact representations, and we address redundancy as well. In particular, we prove that our learned clauses are not subject to any ordering-independent redundancy criterion, e.g. they cannot be subsumed by previously derived clauses.

Finally, the recent calculus $S G G S$ 9] introduces a model based and semantically guided approach. It uses a simpler constraint language and constrained clauses to represent model candidates, and resolves conflicts by repairing the current model assumption through splitting and resolving the constrained clauses in the current representation.

Compared to $S G G S$, we use a more expressive constraint language, our model representation is more explicit, and our search is guided by learning non-redundant clauses.

In the rest of the paper, we first introduce some basic definitions and notions in Section 2, followed by a description of our calculus in Section 3. Section 4 establishes its soundness, while, after introducing some regularity conditions in Section 5 , we provide our key result, namely non-redundant clause learning, in Section 6. We then prove termination in Section 7.

In Section 8, we specify some details on handling constraints, and basic heuristics for a future implementation. We compare our calculus to the existing literature in more details in Section 9. Finally, Section 10 provides a summary and outlines future work.

\section{Preliminaries}

\subsection{Basic Definitions}

We assume the reader is familiar with first-order logic, its syntax, and its semantics. We denote the finite signature by $\Sigma$, the set of predicate symbols by $\operatorname{Pr}$, and the set of function symbols by Fn. For a literal $L,|L|$ denotes the atom contained by $L$.

This paper handles the special case of the Bernays-Schönfinkel fragment, or BS for short. In this case the only functions allowed in the clause normal form are constants, and we call their set the domain and denote it by $\mathcal{D}$.

We denote the set of all first-order atoms over a signature $\Sigma$ and a possibly infinite set of variables $\mathrm{X}$ by $\mathcal{A}_{\Sigma}(\mathrm{X})$. In particular the set of ground atoms is denoted by $\mathcal{A}_{\Sigma}$, a short-hand for $\mathcal{A}_{\Sigma}(\emptyset)$. In general, we denote the ground instances of an expression - a term, literal, or clause - $e$ over the domain $\mathcal{D}$ by the notation $\operatorname{gnd}(e)$.

W.l.o.g., we assume that each independent expression is variable disjoint, and we call a variable fresh if it does not occur in any expression - e.g. clause or clause set - of the current context. 
We consider substitutions in the usual way, and for a substitution $\sigma, \operatorname{dom}(\sigma)$ denotes the domain of $\sigma$, i.e. the finite set of variables with $x \neq x \sigma$, and $\operatorname{rng}(\sigma)$ denotes the range of $\sigma$, i.e. the image of $\operatorname{dom}(\sigma)$ w.r.t. $\sigma$.

We assume the reader is familiar with most general unifiers, and mgu is used to denote the result of unifying two or more expressions or substitutions. We use the short-hand $\exists \sigma=\operatorname{mgu}\left(e_{1}, e_{2}\right)$ to both state the existence of a most general unifier and bind $\sigma$ to one such substitution.

The ordering $\geq$ expresses that the larger expression or substitution is an instance of the smaller one, i.e. $e_{1} \geq e_{2}$ if and only if there is a substitution $\sigma$ such that $e_{1}=e_{2} \sigma$. In the latter case we also say that $e_{2}$ can be matched against $e_{1}$.

We represent a first-order interpretation $I$ with the set $\left\{A \in \mathcal{A}_{\Sigma} \mid I \models A\right\}$. We define satisfiability and semantic consequence as usual.

In particular, we consider the problem of deciding whether a finite clause set $\mathrm{N}$ over a BS language $\Sigma$ without equality is satisfiable. This problem is known to be NEXPTIME-complete [21].

\subsection{Constraints and Constrained Literals}

Next, we provide details about the constraint language we use. Our constraints are essentially implicit generalizations, a constraint language for representing terms and models with exceptions. It has applications in inductive learning, logic programming and term rewriting. For more details see e.g. 11] 20.

The name dismatching constraints was chosen in the spirit of iProver 18, although for our purposes checking satisfiability has to be carried out over the ground instances and thus, the linear-time algorithm of iProver based on matching is not applicable.

While implicit generalizations maintain a list of literals with fresh variables representing exceptions for the literal constrained, dismatching constraints extract the arguments of the literals and represent the restrictions as conjunctions of disequations to allow more simplification and a more compact representation. In particular, we maintain a strict normal form, which already assumes most inexpensive simplifications.

Definition 2.1 (Dismatching Constraint) $A$ dismatching constraint $\pi$ is of the form

$$
\wedge_{i \in \mathcal{I}} \vec{s}_{i} \neq \vec{t}_{i}
$$

where $\mathcal{I}$ is a finite set of indices, and for each $i \in \mathcal{I}, \vec{s}_{i}$ and $\vec{t}_{i}$ are tuples of terms of the same dimension.

Furthermore, we assume that all the left-hand side variables in $\pi$ differ from any right-hand side variable, and for each $i, j \in \mathcal{I}, \vec{t}_{i}$ and $\vec{t}_{j}$ are variable disjoint whenever $i$ differs from $j$.

We further extend the set of constraints with the constants $\top, \perp$ representing the tautological and the unsatisfiable constraint, respectively.

Finally, an atomic constraint $\vec{s} \neq \vec{t}$ occurring in $\pi$ is also called a subconstraint of $\pi$.

To enforce a normal form, we make further assumptions below.

Definition 2.2 (Normal Form) We say a constraint $\pi=\wedge_{i \in \mathcal{I}} \vec{s}_{i} \neq \vec{t}_{i}$ is in normal form iff the following conditions hold: 
(C1) each $\vec{s}_{i}$ contains only variables

(C2) no variable occurs more than once in any left-hand side $\vec{s}_{i}$

A simple consequence of the normal form is that the two sides of any subconstraint $\vec{s} \neq \vec{t}$ are always unifiable, and the induced substitution $\{\vec{s} \leftarrow \vec{t}\}$ is always well-defined and matches the left-hand side against the right-hand side.

Definition 2.3 (Induced Substitutions) The set of induced substitutions of a dismatching constraint $\pi$ in normal form is the set given by

$$
\left\{\left\{\vec{s}_{i} \leftarrow \vec{t}_{i}\right\} \mid i \in \mathcal{I}\right\}
$$

if $\pi=\wedge_{i \in \mathcal{I}} \vec{s}_{i} \neq \vec{t}_{i}$. For $\perp$, we define it as the set containing only the identity, and for $\top$ as the empty set.

We define $\operatorname{lvar}(\pi)$ and $\operatorname{rvar}(\pi)$ as the set of the left-hand side and righthand side variables of some dismatching constraint $\pi$, respectively. Then the semantics for our constraints can be given as below.

Definition 2.4 A solution of a constraint $\pi$ over some variable set $V$, which contains $\operatorname{lvar}(\pi)$ but contains no variable from $\operatorname{rvar}(\pi)$, is a ground substitution $\delta: V \rightarrow \mathcal{D}$ such that no $\vec{t}_{i}$ can be matched against the respective $\vec{s}_{i} \delta$, i.e. no $\vec{s}_{i} \delta$ is an instance of the respective $\vec{t}_{i}$.

In particular, if $\pi=\top$, any such grounding substitution is a solution, and $\pi=\perp$ has no solution at all.

As usual, $\pi$ is called satisfiable and unsatisfiable if it has a solution or no solution, respectively. We note that the notion of satisfiability depends only on $\operatorname{lvar}(\pi)$.

Example 2.5 Consider the domain $\mathcal{D}=\{a, b\}$ and the constraint

$$
\pi=(x, y) \neq(v, v) \wedge y \neq a
$$

Then $\pi$ is satisfiable and the ground substitution $\sigma=\{x \leftarrow a, y \leftarrow b\}$ is the only solution of $\pi$ (over $V=\{x, y\})$.

Remark 2.6 It can be shown that a ground substitution $\delta: V \rightarrow \mathcal{D}$ with $\operatorname{lvar}(\pi) \subseteq V$ is not a solution of $\pi$ if and only if there is an induced substitution $\sigma$ which is more general than $\delta$.

Definition 2.7 Let $\pi$ and $\pi^{\prime}$ denote constraints for which both

- $\operatorname{lvar}(\pi) \cap \operatorname{rvar}\left(\pi^{\prime}\right)=\emptyset$, and

- $\operatorname{lvar}\left(\pi^{\prime}\right) \cap \operatorname{rvar}(\pi)=\emptyset$

hold. Such constraints are called equivalent iff their sets of solutions coincide for any $V$ such that $\operatorname{lvar}(\pi) \cup \operatorname{lvar}\left(\pi^{\prime}\right) \subseteq V$, and both $V \cap \operatorname{rvar}(\pi)=\emptyset$ and $V \cap \operatorname{rvar}\left(\pi^{\prime}\right)=\emptyset$. 


\section{Normal Form Transformation}

Next, we show that any dismatching constraint of the form $\wedge_{i \in \mathcal{I}} \vec{s}_{i} \neq \vec{t}_{i}$ can be normalized in polynomial time. This can be achieved with the rule set below, given as rewriting rules over the subconstraints.

1. $\left(\vec{s}_{1}, a, \vec{s}_{2}\right) \neq\left(\vec{t}_{1}, a, \vec{t}_{2}\right) \Rightarrow\left(\vec{s}_{1}, \vec{s}_{2}\right) \neq\left(\vec{t}_{1}, \vec{t}_{2}\right)$, where $a \in \mathcal{D}$

2. $\left(\vec{s}_{1}, a, \vec{s}_{2}\right) \neq\left(\vec{t}_{1}, b, \vec{t}_{2}\right) \Rightarrow \top$, where $a \neq b \in \mathcal{D}$

3. $\left(\vec{s}_{1}, a, \vec{s}_{2}\right) \neq\left(\vec{t}_{1}, x, \vec{t}_{2}\right) \Rightarrow\left(\vec{s}_{1}, \vec{s}_{2}\right) \neq\left(\overrightarrow{t_{1}}, \overrightarrow{t_{2}}\right) \sigma$, where $a \in \mathcal{D}, \sigma=\{x \leftarrow a\}$

4. ()$\neq() \Rightarrow \perp$

5. $\left(\vec{s}_{1}, x, \vec{s}_{2}, x, \vec{s}_{3}\right) \neq\left(\overrightarrow{t_{1}}, r_{1}, \vec{t}_{2}, r_{2}, \vec{t}_{3}\right) \Rightarrow\left(\vec{s}_{1}, x, \vec{s}_{2}, \vec{s}_{3}\right) \neq\left(\vec{t}_{1}, r_{1}, \vec{t}_{2}, \vec{t}_{3}\right) \sigma$, if $\exists \sigma=\operatorname{mgu}\left(r_{1}, r_{2}\right)$

6. $\left(\vec{s}_{1}, x, \vec{s}_{2}, x, \vec{s}_{3}\right) \neq\left(\vec{t}_{1}, r_{1}, \vec{t}_{2}, r_{2}, \vec{t}_{3}\right) \Rightarrow \top$, if $\nexists \operatorname{mgu}\left(r_{1}, r_{2}\right)$

7. $\vec{s} \neq \vec{t} \Rightarrow \perp$, if $\vec{t}$ can be matched against $\vec{s}$

8. $\left(\vec{s}_{1}, \vec{s}_{2}\right) \neq\left(\overrightarrow{t_{1}}, \overrightarrow{t_{2}}\right) \Rightarrow \overrightarrow{s_{1}} \neq \overrightarrow{t_{1}}$, if $\operatorname{var}\left(\overrightarrow{t_{1}}\right) \cap \operatorname{var}\left(\overrightarrow{t_{2}}\right)=\emptyset$, and $\overrightarrow{t_{2}}$ can be matched against $\vec{s}_{2}$

Where the last rule is considered modulo permutations of positions corresponding to the $\left(\vec{s}_{1}, \vec{s}_{2}\right)$-partitionings.

Example 2.8 Let us normalize the following constraint:

$$
(x, a, y, x) \neq(b, v, w, w) \wedge(x, a, y, x) \neq\left(w_{0}, w_{0}, v_{0}, t_{0}\right)
$$

For the first subconstraint we get

$$
(x, a, y, x) \neq(b, v, w, w) \stackrel{(3)}{\Rightarrow}(x, y, x) \neq(b, w, w) \stackrel{(4)}{\Rightarrow}(x, y) \neq(b, b)
$$

and for the second one

$(x, a, y, x) \neq\left(w_{0}, w_{0}, v_{0}, t_{0}\right) \stackrel{(4)}{\Rightarrow}(x, a, y) \neq\left(w_{0}, w_{0}, v_{0}\right) \stackrel{(3)}{\Rightarrow}(x, y) \neq\left(a, v_{0}\right) \stackrel{(8)}{\Rightarrow} x \neq a$

Thus, the normalized constraint is

$$
(x, y) \neq(b, b) \wedge x \neq a
$$

Applying these rules together with the usual rules for conjunction and the constants $\perp, \top$

1. preserves the variable disjointness conditions of Definition 2.1

2. preserves solutions, i.e. the left-hand side and right-hand side constraints are equivalent

3. transforms $\pi$ into normal form in polynomial time

We note that the rules (7) and (8) are optional, and that (7) is a special case of $(8)$.

Therefore, w.l.o.g. we might assume that the constraints are always in normal form, and the result of any operation is transformed into normal form without explicitly expressing it. We also express it by using the notation $\wedge_{i \in \mathcal{I}} \vec{x}_{i} \neq \vec{t}_{i}$ for dismatching constraints in the rest of the paper. 


\section{Constrained Literals}

Next, we define literals constrained with dismatching constraints in normal form, and give their semantics as sets of ground literals.

Definition 2.9 (Constrained Literal) We call the pair $(L ; \pi)$ of a literal $L$ and a dismatching constraint $\pi$ such that both $\operatorname{lvar}(\pi) \subseteq \operatorname{var}(L)$ and $\operatorname{rvar}(\pi) \cap$ $\operatorname{var}(L)=\emptyset$ hold a constrained literal.

The semantics of constrained literals is given by the following definition of the set of covered literals:

$$
\operatorname{gnd}(L ; \pi)=\left\{L \delta \mid \delta: \operatorname{var}(L) \rightarrow \mathcal{D} \text { s.t. } \forall i \in \mathcal{I}: \nexists \operatorname{mgu}\left(\vec{x}_{i} \delta, \vec{t}_{i}\right)\right\}
$$

where $\pi=\wedge_{i \in \mathcal{I}} \vec{x}_{i} \neq \vec{t}_{i}$. A ground literal $L^{\prime}$ is covered by a constrained literal $(L ; \pi)$ iff $L^{\prime} \in \operatorname{gnd}(L ; \pi)$.

We say that a constrained literal $(L ; \pi)$ is empty if it covers no ground literals, i.e. $\operatorname{gnd}(L ; \pi)$ is empty.

It is easy to see that $(L ; \pi)$ is empty if and only if $\pi$ is unsatisfiable, and that given a solution $\delta$ of $\pi$ over $\operatorname{lvar}(\pi)$, for any extension $\delta^{\prime}$ of $\delta$ to $\operatorname{var}(L)$, $L \delta^{\prime} \in \operatorname{gnd}(L ; \pi)$ holds.

Example 2.10 Let $(L ; \pi)=(P(x, y) ;(x, y) \neq(v, v) \wedge x \neq a \wedge y \neq b)$. Then the set of covered literal over the domain $\mathcal{D}_{2}=\{a, b\}$ is

$$
\operatorname{gnd}(L ; \pi)=\{P(b, a)\}
$$

and if we take $\mathcal{D}_{3}=\{a, b, c\}$ instead, it is

$$
\operatorname{gnd}(L ; \pi)=\{P(b, a), P(c, a), P(b, c)\}
$$

In the rest of the paper we make some further assumptions as common in automated reasoning:

1. Different constrained literals are variable disjoint, unless stated otherwise.

2. Apart from normal form transformations, for any substitution $\sigma$ applied to a constrained literal $(L ; \pi)$, the following always hold unless stated otherwise:

- $\operatorname{dom}(\sigma) \cap \operatorname{rvar}(\pi)=\emptyset$

- $\operatorname{var}(\operatorname{rng}(\sigma)) \cap \operatorname{rvar}(\pi)=\emptyset$

\section{Constrained Clauses}

Occasionally, we have to represent a collection of ground clauses by a constrained clause $(C ; \pi)$. Extending the notations and semantics for constrained literals to constrained clauses is straightforward.

Furthermore, we might use the notation $(C ; \sigma ; \pi)$ for the constrained clause $(C \sigma ; \pi)$, whenever we wish to explicitly separate in notation $C$ and $\sigma$.

We only note that during resolving away literals from $C$, we might get to a state where $\operatorname{lvar}(\pi)$ contains variables not occurring in $C$. See the constrained unit clause

$$
(P(y, z) ;(x, y) \neq(v, v) \wedge(x, z) \neq(w, w))
$$


from Example 8.1 for a demonstration.

For semantic purposes, these free variables are considered existential variables. We assume that such variables are eliminated through instantiation, see Section 8 for further details.

\subsection{Operations on Constrained Literals}

In the context of our calculus, three operations are of significance: conjunction, difference, and checking whether a constrained literal is empty.

Conjunction is used whenever we try to unify two constrained literals, e.g. during learning a new clause via resolution.

Difference is needed when we remove already defined literals ensuring that a new assignment only defines new values.

Finally, emptiness is tested overall in the calculus to ensure that a new assignment indeed defines the value of at least one ground atom.

In the literature checking emptiness also relates to sufficient completeness and negation elimination and it is known to be a co-NP-complete problem 20. in the case of finitely many function symbols and infinite Herbrand universe.

This complexity result also holds for our setting - one might take a binary domain with true and false, and then each atomic constraint with constant righthand side can be seen as clauses with the left-hand side variables as propositional variables, and the emptiness of the whole constraint as the unsatisfiability of this clause set.

In this section, we propose an enumeration-based algorithm to solve emptiness. We believe it will scale better in our context compared to other solutions.

\section{Conjunction}

For two constrained literals $\left(L_{1} ; \pi_{1}\right),\left(L_{2} ; \pi_{2}\right)$ with the same polarity and predicate symbol, we look for a constrained literal $(L ; \pi)$ for which

$$
\operatorname{gnd}(L ; \pi)=\operatorname{gnd}\left(L_{1} ; \pi_{1}\right) \cap \operatorname{gnd}\left(L_{2} ; \pi_{2}\right)
$$

holds. If the two literals are unifiable, such a literal exists. Otherwise, any empty constrained literal can be chosen.

Definition 2.11 (Conjunction) Let as the define and denote the conjunction of two constrained literals $\left(L_{1} ; \pi_{1}\right),\left(L_{2} ; \pi_{2}\right)$ as

$$
\left(L_{1} ; \pi_{1}\right) \wedge\left(L_{2} ; \pi_{2}\right)=\left(L_{1} \sigma ; \pi_{1} \sigma \wedge \pi_{2} \sigma\right)
$$

if $\exists \sigma=\operatorname{mgu}\left(L_{1}, L_{2}\right)$. If the literals are not unifiable, we define it as the empty $\left(L_{1} ; \perp\right)$.

This definition is sound, i.e.

Lemma 2.12 For any unifiable constrained literals $\left(L_{1} ; \pi_{1}\right),\left(L_{2} ; \pi_{2}\right)$,

$$
\operatorname{gnd}\left(L_{1} ; \pi_{1}\right) \cap \operatorname{gnd}\left(L_{2} ; \pi_{2}\right)=\operatorname{gnd}\left(L_{1} \sigma ; \pi_{1} \sigma \wedge \pi_{2} \sigma\right)
$$

holds, where $\sigma=\operatorname{mgu}\left(L_{1}, L_{2}\right)$. 


\section{Proof:}

$(\subseteq)$ : Consider a ground literal from $\operatorname{gnd}\left(L_{1} ; \pi_{1}\right) \cap \operatorname{gnd}\left(L_{2} ; \pi_{2}\right)$, and w.l.o.g. assume it has the form $L_{1} \delta$. Then $L_{1} \delta \geq L_{i}$ holds for both $i=1,2$.

Thus, $\delta=\sigma \epsilon$ for some substitution $\epsilon$. Since $L_{1} \delta \in \operatorname{gnd}\left(L_{i} ; \pi_{i}\right), \pi_{i} \sigma \epsilon$ must be true $(i=1,2)$. But then $L_{1} \delta \geq L_{1} \sigma$, and $L_{1} \delta=\left(L_{1} \sigma\right) \epsilon \in \operatorname{gnd}\left(L_{1} \sigma ; \pi_{1} \sigma \wedge \pi_{2} \sigma\right)$ both hold.

$(\supseteq)$ : Now, assume that $\left(L_{1} \sigma\right) \epsilon$ is a literal from gnd $\left(L_{1} \sigma ; \pi_{1} \sigma \wedge \pi_{2} \sigma\right)$. Then, since $\sigma$ is the most general unifier, we know that $L_{1} \sigma \epsilon \geq L_{i}$ hold for both $i=1$, 2. Furthermore, $\pi_{i} \sigma \epsilon$ is true $(i=1,2)$, and thus, $L_{1} \sigma \epsilon \in \operatorname{gnd}\left(L_{1} ; \pi_{1}\right)$ and $L_{1} \sigma \epsilon=L_{2} \sigma \epsilon \in \operatorname{gnd}\left(L_{2} ; \pi_{2}\right)$. Qed.

We note that the case when no unifier exists is trivial.

Example 2.13 Consider the following constrained literals

- $(L ; \pi)=(P(x, y) ;(x, y) \neq(v, v) \wedge x \neq a \wedge y \neq b)$

- $\left(L^{\prime} ; \pi^{\prime}\right)=(P(z, a) ; z \neq b)$

Then according to the definition above

$$
(L ; \pi) \wedge\left(L^{\prime} ; \pi^{\prime}\right)=(P(z, a) ;(z, a) \neq(v, v) \wedge z \neq a \wedge a \neq b \wedge z \neq b)
$$

which can be simplified to

$$
(P(z, a) ; z \neq a \wedge z \neq b)
$$

This expression is empty over $\mathcal{D}_{2}=\{a, b\}$, and covers exactly the atom $P(c, a)$ over $\mathcal{D}_{3}=\{a, b, c\}$.

\section{Difference}

The difference, or relative difference, $(L ; \pi)$ of two constrained literals $\left(L_{1} ; \pi_{1}\right)$, $\left(L_{2} ; \pi_{2}\right)$ satisfies

$$
\operatorname{gnd}(L ; \pi)=\operatorname{gnd}\left(L_{1} ; \pi_{1}\right)-\operatorname{gnd}\left(L_{2} ; \pi_{2}\right)
$$

Again, if the two literals are unifiable, such a $\pi$ does exist for any finite domain - in the worst case we just add ground constraints to rule out the disallowed atoms. However, this operation might increase the size of $\pi$ exponentially, as demonstrated by the example below.

Example 2.14 Consider the difference

$$
\left(L\left(x_{1}, x_{2}, x_{3}\right) ; \top\right)-\left(L\left(x_{1}, x_{2}, x_{3}\right) ; \wedge_{i=1}^{3} x_{i} \neq a\right)
$$

where $\operatorname{arity}(L)=3$. If $\mathcal{D}_{2}=\{a, b\}$, we might get the still simple expression

$$
\left(L\left(x_{1}, x_{2}, x_{3}\right) ;\left(x_{1}, x_{2}, x_{3}\right) \neq(b, b, b)\right)
$$

However, if $\mathcal{D}_{3}=\{a, b, c\}$, the best we can get is

$$
\left(L\left(x_{1}, x_{2}, x_{3}\right) ;\left(x_{1}, x_{2}, x_{3}\right) \neq(b, b, b) \wedge\left(x_{1}, x_{2}, x_{3}\right) \neq(c, b, b) \wedge \cdots \neq(c, c, c)\right)
$$

It is easy to see that in general, if $|\mathcal{D}|=n$ with $a \in \mathcal{D}$, and $\operatorname{arity}(L)=r$, the size of the resulting constraint is $O\left((n-1)^{r}\right)$. 
Alternatively, one might take a set of disjoint constrained literals describing the difference as follows. First, take the simpler case when $L_{1}$ and $L_{2}$ are the same literal $L$, and consider the difference $\left(L ; \pi_{1}\right)-\left(L ; \pi_{2}\right)$. Assume $\pi_{1}=$ $\wedge_{i \in \mathcal{I}_{1}} \nu_{i}, \pi_{2}=\wedge_{i \in \mathcal{I}_{2}} \eta_{i}$, and $\left\{\sigma_{i} \mid i \in \mathcal{I}_{2}\right\}$ is the set of induced substitutions for $\pi_{2}$. Then, the constrained literal set

$$
\left\{\left(L \sigma_{i} ; \pi_{1} \sigma_{i}\right) \mid i \in \mathcal{I}_{2}\right\}
$$

describes the difference, i.e.

Lemma 2.15

$$
\bigcup_{i \in \mathcal{I}_{2}} \operatorname{gnd}\left(L \sigma_{i} ; \pi_{1} \sigma_{i}\right)=\operatorname{gnd}\left(L ; \pi_{1}\right)-\operatorname{gnd}\left(L ; \pi_{2}\right)
$$

\section{Proof:}

$(\supseteq)$ : Assume $L \delta \in\left(\operatorname{gnd}\left(L ; \pi_{1}\right)-\operatorname{gnd}\left(L ; \pi_{2}\right)\right)$. Since $L \delta \notin \operatorname{gnd}\left(L ; \pi_{2}\right)$, a subconstraint $\eta_{i} \in \pi_{2}$ must be violated, i.e. for some $i \in \mathcal{I}_{2}, \eta_{i} \delta=\perp$.

Then, by the earlier Remark 2.6. $\delta \geq \sigma_{i}$ where $\sigma_{i}$ is the corresponding induced substitution. Thus, $\delta=\sigma_{i} \xi$ for some substitution $\xi$. Finally, since $\pi_{1} \delta=\left(\pi_{1} \sigma_{i}\right) \xi, L \delta \in \operatorname{gnd}\left(L \sigma_{i} ; \pi_{1} \sigma_{i}\right)$ must hold.

$(\subseteq)$ : Now, assume $\left(L \sigma_{i}\right) \xi \in \operatorname{gnd}\left(L \sigma_{i} ; \pi_{1} \sigma_{i}\right)$ for some $i \in \mathcal{I}_{2}$ and grounding substitution $\xi$. Then, we know that $\pi_{1} \sigma_{i} \xi=\top$, and that $\pi_{2} \sigma_{i} \xi=\perp$ since $\eta_{i} \sigma_{i} \xi=\perp$. Thus, $L \sigma_{i} \xi \in\left(\operatorname{gnd}\left(L ; \pi_{1}\right)-\operatorname{gnd}\left(L ; \pi_{2}\right)\right)$. Qed.

However, this set is not pairwise disjoint, and therefore a further step is needed for our purposes.

Lemma 2.16 W.l.o.g. assume $\mathcal{I}_{2}=\{1, \ldots, l\}$, and take

$$
\left\{\left(L \sigma_{i} ; \pi_{1} \sigma_{i} \wedge \eta_{1} \sigma_{i} \wedge \eta_{2} \sigma_{i} \wedge \cdots \wedge \eta_{i-1} \sigma_{i}\right) \mid i=1, \ldots, l\right\}
$$

Then this set still describes the difference and its elements are pairwise disjoint.

Proof: We only prove one inclusion, as the other direction is analogous to the first proof, and disjointness trivially follows form the definition of the set.

$(\supseteq)$ : Assume $L \delta$ is a ground literal from the difference. Thus, $\pi_{1} \delta=\top$ and $\eta_{i} \delta=\perp$ for at least one $i \in \mathcal{I}_{2}$. Let $i$ be the smallest (left-most) such index.

Then, $\delta \geq \sigma_{i}$ must hold along with $\eta_{j} \delta=\top$ for each $j<i$ from $\mathcal{I}_{2}$. Thus, $L \delta \in \operatorname{gnd}\left(L \sigma_{i} ; \pi_{1} \sigma_{i} \wedge \eta_{1} \sigma_{i} \wedge \eta_{2} \sigma_{i} \wedge \cdots \wedge \eta_{i-1} \sigma_{i}\right)$. Qed.

We also note that the above manipulations preserve the variable disjointness of the left-hand and right-hand sides.

Example 2.17 Carrying on with example 2.14 above, we have $\mathcal{I}_{2}=\{1,2,3\}$, $\eta_{i}: x_{i} \neq a$ and $\sigma_{i}=\left\{x_{i} \leftarrow a\right\}$, which gives

$$
\left\{\left(L\left(a, x_{2}, x_{3}\right) ; \top\right),\left(L\left(x_{1}, a, x_{3}\right) ; x_{1} \neq a\right),\left(L\left(x_{1}, x_{2}, a\right) ; x_{1} \neq a \wedge x_{2} \neq a\right)\right\}
$$

as a result.

Let $|\pi|$ denote the size of $\pi$. Then, this operation introduces $O\left(\left|\mathcal{I}_{2}\right|\right)$ atoms with a maximal constraint size of $O\left(\left|\pi_{1}\right|+\left|\pi_{2}\right|\right)$ in general. This gives a total size of $O\left(2\left|\pi^{*}\right|^{2}\right)$ where $\left|\pi^{*}\right|=\max \left\{\left|\pi_{1}\right|,\left|\pi_{2}\right|\right\}$. Clearly, it is independent of the domain size. 
Lemma 2.18 Finally, if $L_{1} \neq L_{2}$, but $\operatorname{mgu}\left(L_{1}, L_{2}\right)=\delta$ exists (otherwise the difference is $\left(L_{1} ; \pi_{1}\right)$ ), and $\vec{u}$ denotes the argument of the top symbol in $L_{1}$, we get the desired set by adding

$$
\left(L_{1} ; \pi_{1} \wedge \vec{u} \neq \vec{u} \delta \rho\right)
$$

to the set $\left(L_{1} \delta ; \pi_{1} \delta\right)-\left(L_{2} \delta ; \pi_{2} \delta\right)$ where the variable renaming $\rho$ introduces fresh variables for the variables in $\vec{u} \delta$.

Proof: The literals in gnd $\left(L_{1} ; \pi_{1}\right)$ can be divided into two disjoint group based on whether they are instances of $L_{2}$ or not.

Those that are no instances of $L_{2}$ are covered by the proposed constrained literal $\left(L_{1} ; \pi_{1} \wedge \vec{u} \neq \vec{u} \delta \rho\right)$. Clearly, each such literal is in the difference.

The common instances are covered by $\left(L_{1} \delta ; \pi_{1} \delta\right)$. From these literals we have to remove those which are covered by $\left(L_{2} ; \pi_{2}\right)$ as well. Clearly, it is enough to compute the difference $\left(L_{1} \delta ; \pi_{1} \delta\right)-\left(L_{2} \delta ; \pi_{2} \delta\right)$.

The resulting set together with $\left(L_{1} ; \pi_{1} \wedge \vec{u} \neq \vec{u} \delta \rho\right)$ covers exactly the elements of the difference. Qed.

It is easy to see that the proofs above hold even if some of the constraints are the constants $\perp$ or $T$, and our definition of induced substitutions in the case of constants supports the proofs.

The disadvantage of the second method is the fragmentation of the constrained literal, especially since after every time we derive a new assignment we have to subtract each unifiable already defined literal.

Remark 2.19 Whenever we compute a difference and get a set of literals as a result, we carry on working with the literals separately.

We could extend our constraint language to handle a set of constrained literals as a single expression. In the literature the corresponding constraints are called disjunctive implicit generalizations, see e.g. [26] for details.

\section{Checking Emptiness}

The problem of deciding whether a constrained literal is empty. As we mentioned before, this is equivalent with the unsatisfiability of the corresponding constraint.

This is in general a co-NP-complete problem 11] 20. Lassez and Marriott proposes an algorithm for computing explicit representation in [20, which can be used for determining emptiness as well. Their algorithm is based on generating disjoint partitions of instances by instantiating a single variable with every possible function symbol at every step.

We also note that emptiness over a finite domain can be encoded as a propositional satisfiability problem, and as such, can be solved with any state-of-the-art CDCL solver.

We propose here an enumeration-based algorithm. Assume $\mathcal{D}=\left\{a_{1}, \ldots, a_{n}\right\}$ is ordered by $>$, and $a_{n}>a_{n-1}>\cdots>a_{0}$. For a constrained literal $(L ; \pi)$ with left-hand side variables $x_{1}, \ldots, x_{k}$, we find a solution $\left(c_{1}, c_{2}, \ldots, c_{k}\right)$ denoting $\left\{x_{j} \leftarrow c_{j} \mid j=1, \ldots, k\right\}$ by enumerating the possible assignments starting with $\left(a_{0}, a_{0}, \ldots, a_{0}\right)$.

If for an intermediate assignment $\left(c_{1}, c_{2}, \ldots, c_{k}\right)$ the subconstraint $\vec{x} \neq \vec{t} \in \pi$ is false, then we increase the value of the right-most position involved in $\vec{x}$. If 
it is already $a_{n}$, we reset it to $a_{0}$ and increase the next involved variable to the left.

If no further increase is possible, there is no solution. If we get a solution for $\vec{x} \neq \vec{t}$, we pick the left-most involved variable which we changed, and reset all non- $\vec{x}$ variables to $a_{0}$.

By repeating the above steps, we either get a solution satisfying $\pi$, or attempt to increase beyond $\left(a_{n}, a_{n}, \ldots, a_{n}\right)$, proving the unsatisfiability of the constraint.

Besides simplicity, this algorithm also has the advantage that the solution might be reusable in operations. We only need to make sure that the solutions for the operands are comparable in the sense that they denote the minimal solutions of the respective constraints w.r.t. the same ordering over the possible assignments.

This can be ensured by ordering the variables based on their left-most occurrence. This way, the solutions of any two non-empty constrained literals with the same predicate symbol are comparable by taking the arguments of the ground literals representing the solutions.

Then, it is enough to consider substitution and adding new subconstraints as primitive operations. Both meet and difference builds upon these steps.

When applying a substitution $\sigma$, we check if the current solution satisfies the positive equality constraints induced by $\sigma$. If yes, then we apply the substitution and keep this solution.

Otherwise, we keep enumerating by always checking the positive conditions first. If we find an assignment satisfying both $\pi$ and $\sigma$, we apply $\sigma$ and save the new solutions. If no solutions to be found, the new constrained literal is empty.

When extending $\pi$ with a new subconstraint $\vec{x} \neq \vec{t}$, we simply continue the enumeration with the current solution and the extended constraint $\pi \wedge \vec{x} \neq \vec{t}$.

\subsection{Model Representation}

\section{Model Candidate}

On the course of this paper, we represent a model candidate, also called a model assumption, as a set $\Gamma$ of constrained literals.

Definition 2.20 A set of constrained literals $\Gamma$ is called consistent if there is no ground atom covered by both a positive and a negative literal from $\Gamma$.

$\Gamma$ is strongly consistent if its elements are pairwise disjoint w.r.t. covering atoms, i.e. for all different $(L ; \pi),\left(L^{\prime} ; \pi^{\prime}\right) \in \Gamma$, gnd $(|L| ; \pi) \cap \operatorname{gnd}\left(\left|L^{\prime}\right| ; \pi^{\prime}\right)=\emptyset$.

We consider only strongly consistent sets in this paper.

Definition 2.21 (Induced Interpretation) The set of positive constrained literals in $\Gamma$ is denoted by $\Gamma^{+}$. Then the first-order interpretation $I_{\Gamma}$ induced by $\Gamma$ is given as

$$
I_{\Gamma}=\bigcup_{(L ; \pi) \in \Gamma^{+}} \operatorname{gnd}(L ; \pi)
$$

\section{Trail}

NRCL attempts to lift the classic CDCL, and as such, it uses a sequence of literals to store the current partial model assumption. 
This trail in our case is a sequence of annotated constrained literals. We retain the notation $\Gamma$, and extend all our definitions and operations for sets of constrained literals to trails as well. We call the elements of $\Gamma$ assignments, as they define truth-values of ground atoms.

Literals in $\Gamma$ are either decision or deduced literals. Decisions are annotated with a unique positive integer, with $(L ; \pi)^{i}$ representing the $i$ th decision in $\Gamma$. Deduced literals are annotated with their reasons, a first-order clause from the current clause set. On the course of the paper, $\alpha$ is used to denote an arbitrary annotation, $C$ to denote a reason clause, and $k, l, i$ to denote decision levels.

We define the value of a ground literal or ground clause true, false, or undefined under $\Gamma$ in the usual sense. In particular, a ground literal $L^{\prime}$ is defined by a constrained literal $(L ; \pi) \in \Gamma$, iff $\left|L^{\prime}\right| \in \operatorname{gnd}(|L| ; \pi)$. If such an $(L ; \pi)$ exists, we also say that $\Gamma$ defines $L^{\prime}$.

A set of ground clauses represented by the constrained clause $(C ; \pi)$ is true or false in $\Gamma$, if all or none of the covered ground instances are true, or false, respectively. The notion of defined by $\Gamma$ can be extended to constrained clauses similarly.

The level $\operatorname{lvl}(L)$ of a ground literal $L$ w.r.t. the trail $\Gamma$ is defined as usual: if $\Gamma$ defines a value for $L$, it is the annotation of the right-most decision on the left of the constrained literal responsible for the assignment, and zero if no decision precedes it.

If $k$ is the level of a literal, we might also say the literal is of level $k$. We call the largest level occurring in a trail the top-level, and also the level of the trail. If no decision occurs in the trail, it is considered 0 .

Following the terminology of SAT solvers, we call a ground clause assertive iff it is false w.r.t. the current trail and contains exactly one top-level literal.

Finally, we say a first-order clause $C$ or a constrained clause $(C \sigma ; \pi)$ is assertive iff $\operatorname{gnd}(C)$, and gnd $(C \sigma ; \pi)$ contains at least one assertive ground clause, respectively.

\section{Induced Abstraction}

Using $\Gamma$ to define truth-values for groups of ground atoms represented by constrained literals can also be seen as providing a propositional abstraction and an abstract partial interpretation.

In this context, our calculus can be seen as a fine-grained abstractionrefinement algorithm, which interleaves refinement and abstract model search, and let the clauses and decision heuristics guide the implicit abstraction and refinement steps.

Below, we provide the related definitions and use these later to define our induced ordering. Beyond this, we do not take any advantage of this connection. Further investigation this direction and utilizing existing results for abstractionrefinement-based procedures is left for future work.

We call a set of positive constrained literals $\Phi$ an abstraction. An abstraction $\Phi$ provides a (partial) partitioning of $\mathcal{A}_{\Sigma}$, and by identifying its elements with propositional atoms, we can assign a propositional abstraction to our clause set N.

These propositional atoms are called abstract atoms. The notions abstract literal and abstract clause are the corresponding syntactic expressions built from 
abstract atoms. We use the abstraction function $\operatorname{def}_{\Phi}$, or simply def, to assign the set of abstract expressions to literals or clauses w.r.t. an abstraction $\Phi$.

Then an abstract interpretation over an abstraction $\Phi$ is simply a propositional interpretation over the corresponding abstract atoms.

If the totality of $\operatorname{def}_{\Phi}$ is needed, we identify uncovered ground atoms with the unique abstract atom $\perp$, and the domain of the interpretation is extended accordingly.

The abstraction $\Phi_{\Gamma}$ induced by $\Gamma$ is defined as

$$
\Phi_{\Gamma}=|\Gamma|=\{(|L| ; \pi) \mid(L ; \pi) \in \Gamma\}
$$

If $\Gamma$ is strongly consistent, $\Phi_{\Gamma}$ is always consistent, i.e. any ground atom is covered by at most one element of $\Phi$.

$\Gamma$ can be seen as defining an abstract interpretation over $\Phi_{\Gamma}$ assigning truthvalues to abstract atoms based on the polarity of the corresponding constrained literals in $\Gamma$, and unde $f$ to the abstract atom $\perp$.

\subsection{Induced Ordering}

In the followings, let $<$ denote a given well-founded total ordering over ground expressions - atoms, literals and clauses. Furthermore, let $\Gamma$ denote a strongly consistent trail.

Definition 2.22 The abstraction function def defined by $\Gamma$ is given as

$$
\operatorname{def}(P)= \begin{cases}(L ; \pi) & \text { if }(L ; \pi) \in \Gamma \text { and }(L ; \pi) \text { defines } P \\ \perp & \text { if no such }(L ; \pi) \in \Gamma \text { exists }\end{cases}
$$

for each $P \in \mathcal{A}_{\Sigma}$.

def can be extended to ground literals and clauses in the usual manner.

Definition 2.23 The precedence ordering $<_{p}^{\Gamma}\left(<_{p}\right)$ defined by $\Gamma$, is the ordering over the constrained literals in $\Gamma$ defined by their position in $\Gamma$, i.e.

$$
\begin{aligned}
& \left(L_{1} ; \pi_{1}\right)<_{p}\left(L_{2} ; \pi_{2}\right) \text { iff } \\
& \qquad \Gamma=\Gamma_{1},\left(L_{1} ; \pi_{1}\right)^{\alpha_{1}}, \Gamma_{2},\left(L_{2} ; \pi_{2}\right)^{\alpha_{2}}, \Gamma_{3}
\end{aligned}
$$

for some $\Gamma_{1}, \Gamma_{2}, \Gamma_{3}$ and annotations $\alpha_{1}, \alpha_{2}$.

We extend the ordering to $\Gamma \cup\{\perp\}$ with $\perp$ as maximal element. Finally, this ordering can be extended to abstract literals and clauses as usual.

Definition 2.24 The ordering $<_{\Gamma}^{\text {atom }}$ induced by $\Gamma$ defined over $\mathcal{A}_{\Sigma}$ is given as follows: $P<_{\Gamma}^{\text {atom }} Q$ iff either

1. $\operatorname{def}(P)<_{p} \operatorname{def}(Q)$, or

2. $\operatorname{def}(P)=\operatorname{def}(Q)$ and $P<Q$

The ordering can be extended to ground literals in the usual way, resulting in the literal ordering $<_{\Gamma}^{\text {lit }}$.

Finally, we extend it to ground clauses: $C<_{\Gamma} C^{\prime}$ iff either

1. $\operatorname{def}(C)<_{p} \operatorname{def}\left(C^{\prime}\right)$, or 
2. $\operatorname{def}(C)=\operatorname{def}\left(C^{\prime}\right)$ and $C\left(<_{\Gamma}^{l i t}\right)_{m u l} C^{\prime}$

where $\left(<_{\Gamma}^{\text {lit }}\right)_{\text {mul }}$ denotes the multiset extension of the literal ordering.

$<_{\Gamma}$ extends the atom and literal orderings, and we call it the ordering induced by $\Gamma$.

Proposition 2.25 $<_{\Gamma}$ is well-defined, total on ground clauses, and a wellfounded ordering.

Proof: It is easy to see that both $<_{p}$ and $\left(<_{\Gamma}^{\text {lit }}\right)_{m u l}$ are well-founded and total orderings over ground clauses. Since $<_{\Gamma}$ is the lexicographical combination of these orderings, $<_{\Gamma}$ inherits these properties. Qed.

Finally, we introduce an easily provable proposition, which is used in the proofs later on.

Proposition 2.26 Let $<$ be an arbitrary well-founded and total ordering over ground clauses, $S$ and $S^{\prime}$ finite sets of ground clauses, and assume there is a function $\gamma: S^{\prime} \rightarrow S$ such that for each $C \in S^{\prime}, C<\gamma(C)$.

Then $S^{\prime}<S$ holds w.r.t. the multiset extension of $<$.

\section{Calculus}

The calculus NRCL attempts to find a model through a series of both arbitrary and deduced assignments. Analogous to the propositional SAT solvers, we apply propagation to find literals implied by existing assignments, and once it is exhausted, we add arbitrary literals, so-called decisions to the trail.

We call this phase conflict search and it ends with either a model of the original clause set, or with finding a clause $C$ with some instances given in the form $(C ; \sigma ; \pi)$ falsified by the current trail. In the latter case, we start conflict resolution and through resolving the current false clause with reason clauses from the trail, we learn a new assertive clause and backtrack to a state where this clause is not yet falsified.

As opposed to propositional SAT solving, where every clause can be considered already exhaustively factorized, in our case some ground instances might be still subject to factorization, and this requires further rules.

The rule Factorize handles this during clause learning. However, the calculus might still reach a state where the right-most literal on the trail is the last decision, the learnable clause is not assertive, but no factorization is possible. When such a state is reached, we simply learn the current candidate for clause learning. To avoid this situation again, we further demand that a new decision should not falsify any clause instance immediately, unless Factorize is applicable.

We call a clause blocking a new decision if adding the decision to the trail would falsify an instance of the clause without allowing Factorize to handle the immediate conflict, see the precise definition below. We note that a clause learned in the above fashion blocks the last decision.

Definition 3.1 We say that a decision $(L ; \pi)$ is blocked by a clause $C \in(N \cup U)$ in $\Gamma$, if $C$ has a ground instance $C \sigma$ with $L_{1}, L_{2} \in C \sigma$ such that for $\Gamma^{\prime}=\Gamma,(L ; \pi)$

- $C \sigma$ is false under $\Gamma^{\prime}$ 
- $(L ; \pi)$ is undefined in $\Gamma$

- $L_{1}$ and $L_{2}$ become false by the decision, i.e. $\neg L_{1}, \neg L_{2} \in \operatorname{gnd}(L ; \pi)$

- $L_{1} \neq L_{2}$

If no such $C$ exists, we say that the decision is not blocked in $\Gamma$.

Example 3.2 Consider $\mathcal{D}=\{a, b, c\}, \Gamma=\left\{(\neg Q(x, y) ; \top)^{1}\right\}$, and

$$
N=\{C: \neg P(x) \vee \neg P(y) \vee Q(x, y), \ldots\}
$$

Then the decisions $(P(x) ; \top),(P(x) ; x \neq c)$ are both blocked by $C$ in $\Gamma$, as witnessed by the ground instance $\neg P(a) \vee \neg P(b) \vee Q(a, b)$.

We give our calculus as a set of rules over so-called states, tuples of the form

$$
(\Gamma ; \mathrm{N} ; \mathrm{U} ; k ; s)
$$

where $\Gamma$ denotes the trail, $\mathrm{N}$ the given clause set, $\mathrm{U}$ the set of learned clauses, $k$ a non-negative integer - unless terminating with Success -, and $s$ a state indicator. The latter can be $\top, \perp$, or a set of clause instances gnd $(C \sigma ; \pi)$ given as $(C ; \sigma ; \pi)$.

$\top$ indicates the conflict search phase, if $k \geq 0$, or that $\Gamma$ defines a model for $\mathrm{N}$, if $k=-1 . \perp$ means the empty clause has been learned, i.e. the unsatisfiability of $\mathrm{N}$ has been established. Finally, an indicator of the form $(C ; \sigma ; \pi)$ represents a set of clause instances falsified by the current trail $\Gamma$, and indicates the conflict resolution phase of our calculus.

Our results extend to any derivation starting from a sound state (see Definition 4.1). Here we propose the initial state

$$
(\epsilon ; \mathrm{N} ; \emptyset ; 0 ; \top)
$$

where $\epsilon$ stands for the empty trail, and $\mathrm{N}$ is the set of first-order clauses being investigated.

Finally, we address a technical question regarding deduced literals and conflict resolution. It is often the case that for a clause $(C \vee L) \in \mathrm{N}$ with $C \sigma$ implying $(L \sigma ; \pi)$ for some $\sigma, \pi$ w.r.t. the current trail $\Gamma$, the involved substitution $\sigma$ substitutes variables not occurring in $L$. See Example 3.3 for a demonstration of this behavior.

Should we save only $(L \sigma ; \pi)$ to the trail, we would lose this part of the assignment. However, during conflict resolution we need the exact clause instances responsible for the assignment. Therefore, to avoid recomputing the relevant substitutions, we save the constrained closure $(L \cdot \sigma ; \pi)$, where $L \cdot \sigma$ is the closure representing $L \sigma$.

This is simply an extension of the existing notation for the sake of clause learning. For all other purposes, $L \cdot \sigma$ is identified with $L \sigma$, and all definitions over constrained literals can be extended to constrained closures accordingly. The literal $L$ is also considered to be a short-hand for $L \cdot \emptyset$. We also note that in our calculus a decision is always considered to have an empty closure.

Below, we provide the rules of our calculus as a state transition system. We note that in the rules $\pi_{1}, \pi_{2}$ is often used as a short-hand for $\pi_{1} \wedge \pi_{2}$, if it is unambiguous. The rules are given as generic as possible. For further details on the applied strategy and technicalities, see Section 5 and Section 8. 


\subsection{Rules for Conflict Search}

\section{Propagate}

$$
(\Gamma ; \mathrm{N} ; \mathrm{U} ; k ; \top) \Rightarrow\left(\Gamma,(L \cdot \sigma ; \pi)^{C \vee L} ; \mathrm{N} ; \mathrm{U} ; k ; \top\right)
$$

if $k \geq 0$, and for some $(C \vee L) \in(\mathrm{N} \cup \mathrm{U}), \sigma$, and $\pi$

- $(C \sigma ; \pi)$ is false under $\Gamma$

- $(L \sigma ; \pi)$ is undefined in $\Gamma$

- $(L \sigma ; \pi)$ is not empty

This rule deduces new literals which have to be true under the current model assumption. The conditions ensure that this step is sound and effective, i.e. each ground literal defined by the added literal is indeed a consequence and at least one such literal exists.

Example 3.3 Let $a, b \in \mathcal{D}, N$ and $U$ arbitrary, $C_{1}, C_{2} \in N$, and the current state

$$
(\Gamma ; N ; U ; 1 ; \top)
$$

where

$$
\Gamma=(P(x, x) ; \top)^{C_{1}},(Q(a, x) ; \top)^{C_{2}},(\neg P(x, y) ;(x, y) \neq(v, v))^{1}
$$

Then, if $C=P(y, b) \vee \neg Q(x, y) \vee R(y)$ is a clause from $N$, Propagate can be applied for $C$, and we might get the state

$$
\left(\Gamma,(R(y) \cdot\{x \leftarrow a\} ; y \neq b)^{C} ; N ; U ; 1 ; \top\right)
$$

Decide

$$
(\Gamma ; \mathrm{N} ; \mathrm{U} ; k ; \top) \Rightarrow\left(\Gamma,(L ; \pi)^{k+1} ; \mathrm{N} ; \mathrm{U} ; k+1 ; \top\right)
$$

if $k \geq 0$, and for some $L, \pi$

- $(L ; \pi)$ is undefined in $\Gamma$

- $(L ; \pi)$ is not blocked in $\Gamma$

- $(L ; \pi)$ is not empty

- $\exists\left(C \vee L^{\prime}\right) \in \mathrm{N}$ such that $|L| \geq\left|L^{\prime}\right|$, i.e. $\exists \delta: L=L^{\prime} \delta$, or $L=\neg L^{\prime} \delta$

Decide adds an assumption to $\Gamma$ which is not blocked by any of the clauses, and which is effective.

We note that the last condition is optional, it does not influence any of our results. This restriction allows earlier termination with Success and keeps the calculus from defining irrelevant ground atoms. After terminating with Success, every undefined ground atom can be considered having arbitrary truth-value, or simply false, the way it is defined in $I_{\Gamma}$.

We also note that blocking only identifies one kind of immediate conflicts, we might still get to an outright conflict if it can be handled with factorization, see Example 3.4 below, and Lemma 5.5 for details. 
Example 3.4 Let $\mathcal{D}=\{a, b, c\}, \Gamma=(P(x, x) ; \top)^{P(x, x)},(Q(x, a) ; \top)^{Q(x, a)}$, and

$$
N=\{P(x, x), Q(x, a), \neg Q(x, y) \vee P(x, y) \vee P(x, y)\}
$$

Then, the decision $(P(x, y) ;(x, y) \neq(v, v))$ is not blocked, yet

$$
(\neg Q(x, y) \vee P(x, y) \vee P(x, y) ;\{y \leftarrow a\} ; x \neq a)
$$

is false w.r.t. $\Gamma,(P(x, y) ;(x, y) \neq(v, v))^{1}$. We note that conflict resolution learns the clause $\neg Q(x, y) \vee P(x, y)$ from this conflict.

We also note that whenever a decision is blocked, we can always pick a stricter unblocked decision, shown below.

Proposition 3.5 For every blocked decision $(L ; \pi)$ and blocking clause $C$, there is a decision $\left(L \sigma ; \pi \sigma, \pi^{\prime}\right)$ for some $\sigma, \pi^{\prime}$ such that it is not blocked by $C$ and it is not empty.

Proof: It is easy to see that any ground literal from $\operatorname{gnd}(L ; \pi)$ satisfies this condition. Qed.

\section{Conflict}

$$
(\Gamma ; \mathrm{N} ; \mathrm{U} ; k ; \top) \Rightarrow(\Gamma ; \mathrm{N} ; \mathrm{U} ; k ;(C ; \sigma ; \pi))
$$

if $k \geq 0$, and for some $\perp \neq C \in(\mathrm{N} \cup \mathrm{U}), \sigma$, and $\pi$

- $(C \sigma ; \pi)$ is false under $\Gamma$

- $(C \sigma ; \pi)$ is not empty

Conflict identifies a set of clause instances contradicting the current model assumption. We also refer to this set as the conflict-set.

Example 3.6 Let $\mathcal{D}=\{a, b, c\}$, and

$$
\begin{aligned}
& N=\{ C_{1}: \neg P(c), C_{2}: \neg P(x) \vee \neg P(y) \vee Q(x, y), \\
&\left.C_{3}: \neg P(y) \vee \neg Q(a, y), C_{4}: \neg Q(x, b) \vee \neg P(x)\right\} \\
& \Gamma=(\neg P(c) ; \top)^{C_{1}},(P(x) ; x \neq c)^{1},(\neg Q(a, y) ; y \neq c)^{C_{3}}
\end{aligned}
$$

Then the following is a valid step:

$(\Gamma ; N ; \emptyset ; 1 ; \top) \stackrel{\text { Conflict }\left(C_{2}\right)}{\Rightarrow}(\Gamma ; N ; \emptyset ; 1 ;(\neg P(x) \vee \neg P(y) \vee Q(x, y) ;\{x \leftarrow a\} ; y \neq c))$

\section{Success}

$$
(\Gamma ; \mathrm{N} ; \mathrm{U} ; k ; \top) \Rightarrow(\Gamma ; \mathrm{N} ; \mathrm{U} ;-1 ; \top)
$$

if $k \geq 0$, and $I_{\Gamma} \models \mathrm{N}$.

We note that the last condition, $I_{\Gamma} \models \mathrm{N}$, can be replaced by demanding that the rules Propagate, Decide and Conflict are exhausted and $\perp \notin(\mathrm{N} \cup \mathrm{U})$.

From this it follows that each ground atom is defined and there is no falsified instance, i.e. every ground clause $C \in \operatorname{gnd}(\mathrm{N} \cup \mathrm{U})$ is true w.r.t. the current trail.

\section{Failure}

if $\perp \in(\mathrm{N} \cup \mathrm{U})$.

$$
(\Gamma ; \mathrm{N} ; \mathrm{U} ; k ; \top) \Rightarrow(\Gamma ; \mathrm{N} ; \mathrm{U} ; 0 ; \perp)
$$

The two terminal rules correspond to the satisfiability and unsatisfiability of the clause set, respectively. Unsatisfiability is detected through learning the empty clause $\perp$. 


\subsection{Rules for Conflict Resolution}

Skip

$$
\left(\Gamma,\left(L^{\prime} \cdot \sigma^{\prime} ; \pi^{\prime}\right)^{C^{\prime}} ; \mathrm{N} ; \mathrm{U} ; k ;(C ; \sigma ; \pi)\right) \Rightarrow(\Gamma ; \mathrm{N} ; \mathrm{U} ; k ;(C ; \sigma ; \pi))
$$

if there is no $L \in C$ such that

- $\exists \eta=\operatorname{mgu}\left(L^{\prime} \sigma^{\prime}, \neg L \sigma\right)$, and

- $\left(C \sigma \eta ; \pi \eta, \pi^{\prime} \eta\right)$ is not empty

Skip drops the right-most literal from the trail during conflict resolution if it is not a decision and it does not contribute to the conflict, i.e. it does not touch any instance of the conflict-set.

\section{Resolve}

$$
\begin{aligned}
& \left(\Gamma,\left(L^{\prime} \cdot \sigma^{\prime} ; \pi^{\prime}\right)^{C^{\prime} \vee L^{\prime}} ; \mathrm{N} ; \mathrm{U} ; k ;(C \vee L ; \sigma ; \pi)\right) \Rightarrow \\
& \left(\Gamma,\left(L^{\prime} \cdot \sigma^{\prime} ; \pi^{\prime}\right)^{C^{\prime} \vee L^{\prime}} ; \mathrm{N} ; \mathrm{U} ; k ;\left(\left(C \vee C^{\prime}\right) \eta_{0} ; \sigma^{*} ; \pi \eta, \pi^{\prime} \eta\right)\right)
\end{aligned}
$$

if for some $L^{\prime}, \sigma, \pi^{\prime}$ and $C^{\prime} \vee L^{\prime}$, and

- $((C \vee L) \sigma ; \pi)$ is not assertive, or $k=0$

- $\exists \eta=\operatorname{mgu}\left(L^{\prime} \sigma^{\prime}, \neg L \sigma\right)$, and let

$-\eta_{0}=\operatorname{mgu}\left(L^{\prime}, \neg L\right)$

$-\sigma^{*}$ such that $\sigma \sigma^{\prime} \eta=\eta_{0} \sigma^{*}$

- $\left((C \vee L) \sigma \eta ; \pi \eta, \pi^{\prime} \eta\right)$ is not empty

We note that keeping $\left.\sigma^{*}\right|_{\operatorname{var}\left(\left(C \vee C^{\prime}\right) \eta_{0}\right)}$ instead of $\sigma^{*}$ is enough for the soundness of the rule and our calculus, as it contains all the relevant information. Furthermore, the existence of $\eta$ implies the existence of $\eta_{0}$ and $\sigma^{*}$.

If the right-most literal in $\Gamma$ is not a decision and is involved in the conflictset, we proceed with resolution. The conditions imply that there are corresponding ground inferences and the new conflict-set is not empty.

Note that dropping the used literal is not desired as the new conflict might still be resolvable with it.

\section{Factorize}

$$
\left(\Gamma, \ell ; \mathrm{N} ; \mathrm{U} ; k ;\left(C \vee L_{1} \vee L_{2} ; \sigma ; \pi\right)\right) \Rightarrow\left(\Gamma, \ell ; \mathrm{N} ; \mathrm{U} ; k ;\left(\left(C \vee L_{1}\right) \eta_{0} ; \sigma^{*} ; \pi \eta\right)\right)
$$

if $\ell=\left(L^{\prime} \cdot \sigma^{\prime} ; \pi^{\prime}\right)^{\alpha}$ for some $L^{\prime}, \sigma^{\prime}, \pi^{\prime}$, and annotation $\alpha$, and

- $\exists \eta=\operatorname{mgu}\left\{L_{1} \sigma, L_{2} \sigma, L^{\prime} \sigma^{\prime}\right\}$, and let

$$
\begin{aligned}
& -\eta_{0}=\operatorname{mgu}\left(L_{1}, L_{2}\right) \\
& -\sigma^{*} \text { such that } \sigma \eta=\eta_{0} \sigma^{*}
\end{aligned}
$$

- $\left(\left(C \vee L_{1}\right) \sigma \eta ; \pi \eta, \pi^{\prime} \eta\right)$ is not empty 
Again, the existence of $\eta$ implies the existence of $\eta_{0}$ and the appropriate $\sigma^{*}$, and keeping $\sigma^{*} \Gamma_{\operatorname{var}\left(\left(C \vee C^{\prime}\right) \eta_{0}\right)}$ is sufficient. We also note that $\alpha$ can be both a reason clause and a decision level.

Factorize factorizes some of the conflicting ground clauses. As in the case of Resolve, the used literal should not be dropped from the trail.

\section{Backjump}

$$
\left(\Gamma_{1}, \Gamma_{2} ; \mathrm{N} ; \mathrm{U} ; k ;(C ; \sigma ; \pi)\right) \Rightarrow\left(\Gamma_{1} ; \mathrm{N} ; \mathrm{U} \cup\{C\} ; k^{\prime} ; \top\right)
$$

if $0 \leq k^{\prime} \leq k, k^{\prime}=\operatorname{lvl}\left(\Gamma_{1}\right)$, and one of the following condition-sets hold:

(1) $k=0$, and $C=\perp$, or

(2) $k>0,(C \sigma ; \pi)$ is assertive, and $C$ has no false instance under $\Gamma_{1}$, or

(3) $k>0$, the right-most element of $\Gamma_{2}$ is the top-level decision, $(C \sigma ; \pi)$ is not assertive, Factorize cannot be applied, and $C$ has no false instance under $\Gamma_{1}$

It is clear that $k^{\prime}=0$ or $k^{\prime}<k$ in case (1) and (2), (3), respectively.

The optimal choice for $k^{\prime}$ the smallest level for which the learned clause can be used in Propagate. Such a $k^{\prime}$ might not always exist for the learned clause $C$, largely due to the instances of $C$ not covered by $(C \sigma ; \pi)$. In these cases the optimal choice for $k^{\prime}$ is the largest level for which $C$ has no false instance. For more details see Section 8.

In case (1), we say that the empty clause $\perp$ is learned. In case (2), we say a new assertive clause is learned, and in case (3) a new blocking clause is learned.

The latter clause is indeed blocking the last decision under some regularity conditions, see Lemma 5.4 for details. We note that case (3) can indeed occur as the following example demonstrates:

\section{Example 3.7 (Learning a blocking clause)}

Consider the clause set

$N=\left\{C_{1}: R(x, x), C_{2}: P(x) \vee \neg Q(x, y), C_{3}: R(x, y) \vee Q(x, y) \vee P(x) \vee P(y)\right\}$

and let $\Gamma=\Gamma^{\prime},(\neg Q(x, y) ; \top)^{C_{2}}$ with

$$
\Gamma^{\prime}=(R(x, x) ; \top)^{C_{1}},(\neg R(x, y) ;(x, y) \neq(v, v))^{1},(\neg P(x) ; \top)^{2}
$$

Then the following is a valid conflict resolution:

$$
\begin{gathered}
(\Gamma ; N ; \emptyset ; 2 ;(R(x, y) \vee Q(x, y) \vee P(x) \vee P(y) ; \emptyset ;(x, y) \neq(v, v))) \stackrel{\text { Resolve }}{\Rightarrow} \\
(\Gamma ; N ; \emptyset ; 2 ;(R(x, y) \vee P(x) \vee P(x) \vee P(y) ; \emptyset ;(x, y) \neq(v, v))) \stackrel{\text { Skip }}{\Rightarrow} \\
\left(\Gamma^{\prime} ; N ; \emptyset ; 2 ;(R(x, y) \vee P(x) \vee P(x) \vee P(y) ; \emptyset ;(x, y) \neq(v, v))\right) \stackrel{\text { Factorize }}{\Rightarrow} \\
\left(\Gamma^{\prime} ; N ; \emptyset ; 2 ;(R(x, y) \vee P(x) \vee P(y) ; \emptyset ;(x, y) \neq(v, v))\right) \stackrel{\text { Backjump }(3)}{\Rightarrow} \\
\left((R(x, x) ; \top)^{C_{1}},(\neg R(x, y) ;(x, y) \neq(v, v))^{1} ; N ;\{R(x, y) \vee P(x) \vee P(y)\} ; 1 ; \top\right)
\end{gathered}
$$


Remark 3.8 We also wish to note that the current formulation of the calculus handles blocking decisions and learning blocking clauses asymmetrically in the following sense.

Let $\mathcal{D}=\{a, b, c\}, N=\left\{P(x, x), Q(x, a), \neg Q(x, y) \vee P(x, y) \vee P\left(x^{\prime}, y\right)\right\}$, and

$$
\Gamma=(P(x, x) ; \top)^{P(x, x)},(Q(x, a) ; \top)^{Q(x, a)}
$$

Then the decision $(\neg P(x, y) ;(x, y) \neq(z, z))$ is blocked by $\neg Q(x, y) \vee P(x, y) \vee$ $P\left(x^{\prime}, y\right)$. We could use factorization and learn $(\neg Q(x, y) \vee P(x, y))$, but instead we rather throw away the decision candidate and try another.

On the other hand, if in some regular run (see Definition 5.2) a conflict state of the form

$$
\left(\Gamma^{\prime}, \ell^{k} ; N^{\prime} ; U^{\prime} ; k ;\left(\neg Q(x, y) \vee P(x, y) \vee P\left(x^{\prime}, y\right) ;\{y \leftarrow a\} ; x \neq a \wedge x^{\prime} \neq a\right)\right)
$$

with $\ell=(\neg P(x, y) ;(x, y) \neq(z, z))$ arises, we choose Factorize over learning a blocking clause outright - there is indeed a blocking instance -, and learn the assertive and not-blocking $\neg Q(x, y) \vee P(x, y)$ in the end.

\subsection{Example}

Example 3.9 Consider $\mathcal{D}=\{a, b, c\}$ and the finite clause set

$$
N=\left\{\begin{array}{lll}
C_{1}: \neg P(c), & & C_{2}: \neg P(x) \vee \neg P(y) \vee Q(x, y), \\
& C_{3}: \neg P(y) \vee \neg Q(a, y), & C_{4}: \neg Q(x, b) \vee \neg P(x)
\end{array}\right.
$$

Then the following NRCL derivation constructs a model for $N$ over $\mathcal{D}$.

$$
\begin{aligned}
& (\epsilon ; N ; \emptyset ; 0 ; \top) \stackrel{\text { Propagate }}{\Rightarrow}\left((\neg P(c) ; \top)^{C_{1}} ; N ; \emptyset ; 0 ; \top\right) \stackrel{\text { Decide }}{\Rightarrow} \\
& \left((\neg P(c) ; \top)^{C_{1}},(P(x) ; x \neq c)^{1} ; N ; \emptyset ; 1 ; \top\right) \stackrel{\text { Propagate }}{\Rightarrow} \\
& \left((\neg P(c) ; \top)^{C_{1}},(P(x) ; x \neq c)^{1},(\neg Q(a, y) ; y \neq c)^{C_{3}} ; N ; \emptyset ; 1 ; \top\right) \stackrel{\text { Conflict }_{2}}{\Rightarrow} \\
& (\ldots ; N ; \emptyset ; 1 ;(\neg P(x) \vee \neg P(y) \vee Q(x, y) ;\{x \leftarrow a\} ; y \neq c)) \\
& \stackrel{\text { Resolve }}{\Rightarrow}\left(\left(\ldots,(\neg Q(a, y) ; y \neq c)^{C_{3}} ; N ; \emptyset ; 1 ;(\neg P(a) \vee \neg P(y) \vee \neg P(y) ; \emptyset ; y \neq c)\right)\right. \\
& \stackrel{S k i p}{\Rightarrow}\left(\left((\neg P(c) ; \top)^{C_{1}},(P(x) ; x \neq c)^{1} ; N ; \emptyset ; 1 ;(\neg P(a) \vee \neg P(y) \vee \neg P(y) ; \emptyset ; y \neq c)\right)\right. \\
& \stackrel{\text { Factorize }}{\Rightarrow}\left(\left((\neg P(c) ; \top)^{C_{1}},(P(x) ; x \neq c)^{1} ; N ; \emptyset ; 1 ;(\neg P(a) \vee \neg P(y) ; \emptyset ; y \neq c)\right)\right. \\
& \stackrel{\text { Factorize }}{\Rightarrow}\left((\neg P(c) ; \top)^{C_{1}},(P(x) ; x \neq c)^{1} ; ; N ; \emptyset ; 1 ;(\neg P(a) ; \emptyset ; \top)\right) \stackrel{\text { Backjump }(2)}{\Rightarrow} \\
& \left((\neg P(c) ; \top)^{C_{1}} ; N ; U_{1} ; 0 ; \top\right) \stackrel{\text { Propagate }}{\Rightarrow}\left((\neg P(c) ; \top)^{C_{1}},(\neg P(a) ; \top)^{C_{5}} ; N ; U_{1} ; 0 ; \top\right) \\
& \stackrel{\text { Decide }}{\Rightarrow}\left((\neg P(c) ; \top)^{C_{1}},(\neg P(a) ; \top)^{C_{5}},(P(b) ; \top)^{1} ; N ; U_{1} ; 1 ; \top\right) \stackrel{\text { Propagate }}{\Rightarrow} \\
& \left((\neg P(c) ; \top)^{C_{1}},(\neg P(a) ; \top)^{C_{5}},(P(b) ; \top)^{1},\left(Q(x, y) \cdot \sigma_{1} ; \top\right)^{C_{2}} ; N ; U_{1} ; 1 ; \top\right) \stackrel{\text { Conflict } C_{4}}{\Rightarrow}
\end{aligned}
$$


Where $\sigma_{1}=\{x \leftarrow b, y \leftarrow b\}$.

$$
\begin{aligned}
& \left(\ldots,(P(b) ; \top)^{1},\left(Q(x, y) \cdot \sigma_{1} ; \top\right)^{C_{2}} ; N ; U_{1} ; 1 ;(\neg Q(x, b) \vee \neg P(x) ;\{x \leftarrow b\} ; \top)\right) \stackrel{\text { Resolve }}{\Rightarrow} \\
& \left(\ldots,(P(b) ; \top)^{1},\left(Q(x, y) \cdot \sigma_{1} ; \top\right)^{C_{2}} ; N ; U_{1} ; 1 ;(\neg P(x) \vee \neg P(x) \vee \neg P(b) ;\{x \leftarrow b\} ; \top)\right) \stackrel{\text { Skip }}{\Rightarrow} \\
& \left(\ldots,(\neg P(a) ; \top)^{C_{5}},(P(b) ; \top)^{1} ; N ; U_{1} ; 1 ;(\neg P(x) \vee \neg P(x) \vee \neg P(b) ;\{x \leftarrow b\} ; \top)\right) \stackrel{\text { Factorize }}{\Rightarrow} \\
& \left((\neg P(c) ; \top)^{C_{1}},(\neg P(a) ; \top)^{C_{5}},(P(b) ; \top)^{1} ; N ; U_{1} ; 1 ;(\neg P(x) \vee \neg P(b) ;\{x \leftarrow b\} ; \top)\right) \stackrel{\text { Factorize }}{\Rightarrow} \\
& \quad\left((\neg P(c) ; \top)^{C_{1}},(\neg P(a) ; \top)^{C_{5}},(P(b) ; \top)^{1} ; N ; U_{1} ; 1 ;(\neg P(b) ; \emptyset ; \top)\right) \stackrel{\text { Backjump }(2)}{\Rightarrow} \\
& \quad\left((\neg P(c) ; \top)^{C_{1}},(\neg P(a) ; \top)^{C_{5}} ; N ; U_{1} \cup\{\neg P(b)\} ; 0 ; \top\right) \stackrel{\text { Propagate }}{\Rightarrow} \\
& \quad\left((\neg P(c) ; \top)^{C_{1}},(\neg P(a) ; \top)^{C_{5}},(\neg P(b) ; \top)^{C_{6}} ; N ; U_{2} ; 0 ; \top\right) \stackrel{\text { Decide }}{\Rightarrow}
\end{aligned}
$$

Where $U_{2}=U_{1} \cup\left\{C_{6}: \neg P(b)\right\}$.

$$
\begin{gathered}
\left((\neg P(c) ; \top)^{C_{1}},(\neg P(a) ; \top)^{C_{5}},(\neg P(b) ; \top)^{C_{6}},(Q(x, y) ; \top)^{1} ; N ; U_{2} ; 1 ; \top\right) \stackrel{\text { Success }}{\Rightarrow} \\
\quad\left((\neg P(c) ; \top)^{C_{1}},(\neg P(a) ; \top)^{C_{5}},(\neg P(b) ; \top)^{C_{6}},(Q(x, y) ; \top)^{1} ; N ; U_{2} ;-1 ; \top\right)
\end{gathered}
$$

\section{Soundness}

Now, we show soundness. The following state invariant defines a consistency notion for states.

Definition 4.1 A state $(\Gamma ; N ; U ; k ; s)$ is sound if and only if the followings hold:

1. $\Gamma$ is a consistent sequence of constrained literals

2. $\Gamma$ is well-formed, i.e.

(a) if $k \geq 0$ then $\Gamma$ contains exactly $k$ decisions

(b) for each $i$ from $1,2, \ldots, k$, there is a unique $(L ; \pi)^{i} \in \Gamma$

(c) the decisions occur in $\Gamma$ in the order of their levels

(d) for each decomposition $\Gamma=\Gamma_{1},(L ; \pi)^{i}, \Gamma_{2} ;(L, \pi)^{i}$ satisfies the conditions of Decide w.r.t. $\Gamma_{1}, N$, and $U$

(e) for each decomposition $\Gamma=\Gamma_{1},(L \cdot \sigma ; \pi)^{C \vee L}, \Gamma_{2} ;(C \sigma ; \pi)$ is false under $\Gamma_{1}$, and $(L \sigma ; \pi)$ satisfies the conditions for Propagate w.r.t. $\Gamma_{1}$ and $C \vee L$

3. $N \models U$

4. $s=\perp$ implies $\perp \in N \cup U$

5. $k=-1$ implies $I_{\Gamma} \models N$

6. if $s=(C ; \sigma ; \pi)$ then $(C \sigma ; \pi)$ is false under $\Gamma, N \models C$, and $(C \sigma ; \pi)$ is not empty.

A rule is called sound iff it preserves the soundness of its left-hand side state. 
It is easy to see that the initial state $(\epsilon ; \mathrm{N} ; \emptyset ; 0 ; \top)$ is always sound. Furthermore, soundness is an invariant, since each rule preserves this property, as proven below.

Theorem 4.2 The rules of NRCL are sound.

Proof: The soundness of Propagate, Decide, Conflict, and the terminal rules Failure and Success is straightforward to prove from the definitions themselves, and therefore, we entrust it to the reader.

In the case of Skip, dropping the right-most literal $\left(L^{\prime} \cdot \sigma^{\prime} ; \pi^{\prime}\right)^{C^{\prime}}$ from $\Gamma$ does preserve the well-formedness and consistency properties of $\Gamma$. N $\models U$ remains unchanged and the rest of the conditions are irrelevant in this case, except for the last one.

Now, assume the last property does not hold after applying Skip. It is only possible if some ground clause $C^{\prime \prime}$ from $\operatorname{gnd}(C \sigma ; \pi)$ were false under $\Gamma,\left(L^{\prime}\right.$. $\left.\sigma^{\prime} ; \pi^{\prime}\right)^{C^{\prime}}$, but is undefined under $\Gamma$. Thus, $\left(L^{\prime} \sigma^{\prime} ; \pi^{\prime}\right)$ must have made it false, and therefore, for some $\delta$ and $L^{\prime \prime} \in C^{\prime \prime}, L^{\prime \prime}=\neg L^{\prime} \sigma^{\prime} \delta$ and $\pi^{\prime} \delta$ is true.

Let $L$ be the literal in $C$ corresponding to $L^{\prime \prime}$. Then, the most general unifier $\eta$ of $\neg L \sigma$ and $L^{\prime} \sigma^{\prime}$ must exist and $C^{\prime \prime} \in \operatorname{gnd}\left(C \sigma \eta ; \pi \eta, \pi^{\prime} \eta\right)$, which is therefore not empty. This violates the preconditions of Skip, a contradiction.

For Resolve, it is enough to see that the new clause is a consequence of $\mathrm{N}$, and the new state indicator $\left(\left(C \vee C^{\prime}\right) \eta_{0} ; \sigma^{*} ; \pi \eta, \pi^{\prime} \eta\right)$ is unsatisfiable under $\Gamma$, using the notations of the definition for Resolve.

The first claim follows from the soundness of the left-hand side and from the soundness of resolution. As for the second claim, we make the following observations:

- $\left(C \vee C^{\prime}\right) \eta_{0} \sigma^{*}=\left(C^{\prime} \vee C\right) \sigma \sigma^{\prime} \eta$

- Each instance from gnd $\left(C^{\prime} \sigma^{\prime} \eta ; \pi^{\prime} \eta\right)$ is false under the current trail, as per the well-formedness conditions for derived literals.

- Each instance from gnd $(C \sigma \eta ; \pi \eta)$ is false under the trail by the soundness of the left-hand side.

From these it follows that each ground clause from gnd $\left(C \vee C^{\prime} ; \sigma \sigma^{\prime} \eta ; \pi \eta, \pi^{\prime} \eta\right)$ is false under the current trail.

The soundness of Factorize can be proven analogously, and the proof for Backjump is straightforward. We entrust them to the reader. Qed.

Next, we define runs, i.e. sound derivations in our calculus.

Definition 4.3 A run (from a clause set $N$ ) is a sequence of states such that each subsequent state is derived with a rule from the previous one, and the initial state is a sound state (with $N$ as the original clause set).

A direct consequence of Theorem 4.2 is that each state in a run is sound, and in particular, for each conflict resolution state $(\Gamma ; \mathrm{N} ; \mathrm{U} ; k ;(C ; \sigma ; \pi))$, each ground clause from $\operatorname{gnd}(C \sigma ; \pi)$ is false w.r.t. $\Gamma$.

Theorem 4.4 (Soundness) The calculus NRCL is sound, i.e. if a run terminates with the Failure, or Success rules, then the starting set $N$ is unsatisfiable, and satisfiable, respectively. Furthermore, in the latter case the trail upon termination defines a model of $N$.

Proof: It follows immediately from the definitions and Theorem 4.2 Qed. 


\section{Regular Runs}

In this section, we define a strategy for NRCL in the form of regular runs, which is sufficient to prove both non-redundant clause learning, and termination in the later sections.

Definition 5.1 A sound state $(\Gamma ; N ; U ; k ; s)$ is regular iff the following hold:

- If $\Gamma=\Gamma^{\prime},(L \cdot \sigma ; \pi)^{\alpha}$, then no clause from $N \cup U$ is false w.r.t. $\Gamma^{\prime}$.

- For all decomposition $\Gamma=\Gamma_{1},(L ; \pi)^{i}, \Gamma_{2}$ with decision $(L ; \pi)^{i}$, Propagate is exhausted w.r.t. $\Gamma_{1}$ and $N \cup U$.

We note that the last assignment on the trail might still make some clauses false, and the initial state $(\epsilon ; \mathrm{N} ; \emptyset ; 0 ; \top)$ is always regular.

Definition 5.2 We call a run regular iff the following holds:

- The starting state is regular.

- During conflict search, rules are always applied in this order exhaustively: terminal rules, Conflict, Propagate, Decide. (Or Failure, Conflict, Propagate, Decide, Success, if we test success through exhausted conflict search.)

- In conflict resolution Backjump is always applied as soon as possible, and it backtracks to a regular state.

Lemma 5.3 Regular runs preserve regularity, i.e. all state in a run is regular.

Proof: It follows from the definitions, we only note that backjumping to a state which is regular w.r.t. the new learned clause set as well is always possible. If nothing else, the empty trail is always a valid choice. Qed.

The backtrack-level proposed in the proof above is not practical, of course. For more details on a more accurate backjumping to a regular state see Section 8 .

Below, we show some useful properties of regular runs.

Lemma 5.4 In a regular run the following hold:

(1) For any deduced literal $(L \cdot \sigma ; \pi)^{C \vee L}$ of level $k$ on the trail, each ground clause in $\operatorname{gnd}((C \vee L) \sigma ; \pi)$ contains at least two literals of level $k$.

(2) If $(C ; \sigma ; \pi)$ represents false clauses in some conflict state, then each ground clause in $\operatorname{gnd}(C \sigma ; \pi)$ contains at least two top-level literals, if the state is the result of an application of Conflict, and at least one top-level literal otherwise.

(3) If a clause $C$ is learned according to the case Backjump-(3), then it blocks the former top-level decision.

Proof: First, assume $(L \cdot \sigma ; \pi)$ is a deduced literal and it was implied by $(C \vee$ $L ; \sigma ; \pi)$ w.r.t. $\Gamma$ which was the current trail before the corresponding application of Propagate. 
Let $k$ be the level of the right-most decision in $\Gamma$, and $C^{\prime} \vee L^{\prime}$ a ground clause from gnd $((C \vee L) \sigma ; \pi)$ such that $L^{\prime}$ corresponds to $L$. Then $L^{\prime}$ is of level $k$, of course.

Furthermore, if no other literal in $C^{\prime}$ is of level $k, C^{\prime} \vee L^{\prime}$ would have implied $L^{\prime}$ before the last decision, which contradicts the exhaustive application of Propagate. Thus, $C^{\prime} \vee L^{\prime}$ must contain at least two literals of level $k$.

Second, since conflicts are found immediately, any conflicting non-empty ground clause $C^{\prime}$ must contain at least one top-level literal. A conflicting ground clause with a single top-level literal, however, would contradict the exhaustive application of Propagate. Thus, after applying Conflict, all ground clause in the conflict-set contains at least two top-level literals. It only remains to show that the rules Resolve, Skip, and Factorize preserve the weaker property of having at least one top-level literals. Obviously, e.g. Factorize can break the stronger property.

We only prove this for Resolve, the rest can be shown similarly. Assume that at an application of Resolve $\left(L^{\prime} \cdot \sigma^{\prime} ; \pi^{\prime}\right)^{C^{\prime} \vee L^{\prime}}$ is the involved deduced literal, $(C \vee \neg L ; \sigma ; \pi)$ represents the false clauses before, and $\left(\left(C \vee C^{\prime}\right) \eta_{0} ; \sigma^{*} ; \pi \eta, \pi^{\prime} \eta\right)$ after applying the rule, where $\eta=\operatorname{mgu}\left(L^{\prime} \sigma^{\prime}, L \sigma\right), \eta_{0}=\operatorname{mgu}\left(L^{\prime}, L\right)$, and $\sigma^{*}$ such that $\eta_{0} \sigma^{*}=\sigma \sigma^{\prime} \eta$.

It is easy to see that for every ground clause

$$
\left(C_{0} \vee C_{0}^{\prime}\right) \in \operatorname{gnd}\left(\left(C \vee C^{\prime}\right) \sigma \sigma^{\prime} \eta ; \pi \eta, \pi^{\prime} \eta\right)
$$

there are corresponding ground clauses $\left(C_{0}^{\prime} \vee L_{0}^{\prime}\right) \in \operatorname{gnd}\left(\left(C^{\prime} \vee L^{\prime}\right) \sigma^{\prime} \eta ; \pi^{\prime} \eta\right)$ and $\left(C_{0} \vee \neg L_{0}\right) \in \operatorname{gnd}((C \vee \neg L) \sigma \eta ; \pi \eta)$ whose resolvent is exactly $\left(C_{0} \vee C_{0}^{\prime}\right)$, and $L_{0}$, $L_{0}^{\prime}$ correspond to $L$ and $L^{\prime}$, respectively, and $L_{0}=L_{0}^{\prime}$.

Then, by the first claim of this lemma, $C_{0}^{\prime}$ must contain at least one top-level literals, and so does $C_{0} \vee C_{0}^{\prime}$.

Finally, assume $C$ is learned when case (3) of Backjump is applied to the state

$$
\left(\Gamma,(L ; \pi)^{k} ; \mathrm{N} ; \mathrm{U} ; k ;(C ; \sigma ; \pi)\right)
$$

Now, let $\left(C^{\prime} \vee L_{1}^{\prime} \vee \cdots \vee L_{s}^{\prime}\right) \in \operatorname{gnd}(C \sigma ; \pi)$ an arbitrary ground clause, where $L_{1}^{\prime}, \ldots, L_{s}^{\prime}$ denotes the top-level literals of the clause.

By $(2), s \geq 1$, and, since $(C \sigma ; \pi)$ has no assertive clause, even $s \geq 2$ must hold. We also know that Factorize was not applicable, thus, for any $i \neq j$ from $1, \ldots, s, L_{i} \neq L_{j}$ holds. Thus, $C$ blocks the decision $(L ; \pi)$ w.r.t. $\Gamma$, as witnessed by the ground clause above. Qed.

It can be also shown that if there is an immediate conflict after a decision in a regular run, Factorize is applied next.

\section{Lemma 5.5 Assume}

$$
\stackrel{\text { Decide }}{\Rightarrow}\left(\Gamma,(L ; \pi)^{k} ; N ; U ; k ; \top\right) \stackrel{\text { Conflict }}{\Rightarrow}\left(\Gamma,(L ; \pi)^{k} ; N ; U ; k ;\left(C ; \sigma^{\prime} ; \pi^{\prime}\right)\right)
$$

is a valid subderivation in a regular run. Then Factorize, and only Factorize, is applicable to the conflict state $\left(\Gamma,(L ; \pi)^{k} ; N ; U ; k ;\left(C ; \sigma^{\prime} ; \pi^{\prime}\right)\right)$.

Proof: Obviously, Resolve and Skip cannot be applied. Furthermore, if case Backjump-(3) were applicable, there would be a ground clause in $\operatorname{gnd}\left(C \sigma^{\prime} ; \pi^{\prime}\right)$ blocking the last decision, a contradiction. 
Also, there cannot be any ground clause in $\operatorname{gnd}\left(C \sigma^{\prime} ; \pi^{\prime}\right)$ with a single toplevel literal, since otherwise Propagate would not have been applied exhaustively before the decision. And $C=\perp$ cannot hold either, as otherwise Failure should have been applied earlier. Thus, the other cases of Backjump do not apply either.

Finally, let $C_{0}$ a ground clause from gnd $\left(C \sigma^{\prime} ; \pi^{\prime}\right)$. This clause exists, and must contain at least two top-level literals, see Lemma 5.4(2). These literals are falsified by the last decision, and do not block the decision.

Let $L_{0}, K_{0}$ two such literals and $C_{0}=C_{0}^{\prime} \vee L_{0} \vee K_{0}$. Then these literals are equal, and the corresponding literals $L_{1}, K_{1}$ in $C \sigma$ are unifiable.

Then Factorize is applicable unifying $L_{1}$ and $K_{1}$, and $C_{0}^{\prime} \vee L_{0}$ can be used to prove the non-emptiness condition. Qed.

\section{Redundancy}

We define redundancy w.r.t. the induced ordering $<_{\Gamma}$ in the standard way:

Definition 6.1 A ground clause $C$ is redundant w.r.t. a ground clause set N (and $<_{\Gamma}$ ) iff

$$
C \in N \text {, or } \exists S \subseteq N^{<{ }_{\Gamma} C}: S \models C
$$

A first-order clause $C$ is redundant w.r.t. the first-order clause set $\mathrm{N}$ (and $<_{\Gamma}$ ) iff

$$
\forall C^{\prime} \in \operatorname{gnd}(C): C^{\prime} \text { is redundant w.r.t. } \operatorname{gnd}(N)
$$

If redundancy does not hold, we call the corresponding clause non-redundant, or irredundant.

\subsection{Learning Non-Redundant Clauses}

First, we show that each learned clause is non-redundant w.r.t. the current clause set and induced ordering.

The most important consequence of this theorem that checking the learned clauses for redundancy criterions which are independent from the concrete induced orderings can be spared.

Such admissible criterions include subsumption, subsumption resolution and tautologies, as it is shown in the next subsection.

Theorem 6.2 (Non-redundant Clause Learning) Let $\Gamma$ denote the trail at a conflict in a regular run, $<_{\Gamma}$ the induced ordering, and assume the clause $C$ is learned via the Backjump rule, and let $N$ and $U$ be the starting clause set and the set of learned clauses before the conflict, respectively.

Then, $C$ is not redundant w.r.t. $N \cup U$ and $<_{\Gamma}$.

Proof: Assume the first and last state in conflict resolution is

$$
\left(\Gamma ; \mathrm{N} ; \mathrm{U} ; k ;\left(C_{0} ; \sigma_{0} ; \pi_{0}\right)\right) \Rightarrow^{*}\left(\Gamma^{\prime} ; \mathrm{N} ; \mathrm{U} ; k ;\left(C ; \sigma_{1} ; \pi_{1}\right)\right)
$$

By soundness, $\mathrm{N} \cup \mathrm{U} \models C$ and each $C^{\prime} \in \operatorname{gnd}\left(C \sigma_{1} ; \pi_{1}\right)$ is false w.r.t. both $\Gamma^{\prime}$ and $\Gamma$. 
Now let $C^{\prime} \in \operatorname{gnd}\left(C \sigma_{1} ; \pi_{1}\right)$ and assume there is an $S \subset \operatorname{gnd}(\mathrm{N} \cup \mathrm{U})$ such that $S \models C^{\prime}$ and $S<_{\Gamma} C^{\prime}$. Because of $S<_{\Gamma} C^{\prime}$, each $C^{\prime \prime} \in S$ has a defined truthvalue w.r.t. $\Gamma$. If all $C^{\prime \prime} \in S$ is true, then, by $S \models C^{\prime}$, so is $C^{\prime}$, a contradiction.

Thus, let $C^{\prime \prime} \in S$ arbitrary such that $C^{\prime \prime}$ is false under $\Gamma$. We distinguish two cases whether $\Gamma^{\prime}$ is a strict subset of $\Gamma$, or equal to it.

First, if $\Gamma^{\prime} \neq \Gamma$, at least one Skip had to be used, and $C^{\prime}$ contains no literal covered by the right-most literal of $\Gamma$. Neither does $C^{\prime \prime}$, since $C^{\prime \prime}<_{\Gamma} C^{\prime}$. But then, $C^{\prime \prime}$ has a defined truth-value and it can only be true, as otherwise an earlier conflict detection would have been possible. A contradiction.

Second, assume $\Gamma^{\prime}=\Gamma$. If the right-most literal is a decision, no false clause from gnd $\left(C_{0} \sigma_{0} ; \pi_{0}\right)$ blocks this decision, and Factorize had to be applied several times followed by an application of case (2) of Backjump. (See also Lemma 5.5 on immediate conflicts.)

Let now $C^{\prime}$ such that it contains only a single top-level literal. Since case (2) of Backjump was used, such a clause from gnd $\left(C \sigma_{1} ; \pi_{1}\right)$ exists. Since $C^{\prime \prime}$ is false and it was undefined before, it contains some top-level literals.

Since it was not a subject of Propagate before the right-most decision, it has to contain at least two such literals. But $C^{\prime}$ contains only one, and therefore $\operatorname{def}\left(C^{\prime}\right)<_{p} \operatorname{def}\left(C^{\prime \prime}\right)$ and $C^{\prime}<_{\Gamma} C^{\prime \prime}$ must hold, a contradiction.

Finally, if $\Gamma=\Gamma^{\prime}$ and the right-most literal is not a decision, the last rule had to be Backjump (case 1 or 2), and the same argumentation holds: If an assertive clause is learned, let $C^{\prime}$ an instance from $\operatorname{gnd}\left(C \sigma_{1} ; \pi_{1}\right)$ such that it contains only a single top-level literal. However, $C^{\prime \prime}$ must contain at least two top-level literals, which again leads to $C^{\prime}<_{\Gamma} C^{\prime \prime}$, a contradiction. If $C=\perp$ is learned, it is smaller than any non-empty clause, and due to regularity, $\perp$ is a newly learned clause. Qed.

\subsection{Admissible Redundancies}

Next, we show that the classic redundancy criterions tautology, strict subsumption, and subsumption resolution are admissible redundancies in NRCL, i.e. the clauses these rules remove are indeed redundant w.r.t. any induced ordering.

Proposition 6.3 (Tautology) Let $C$ a clause and $N$ an arbitrary clause set.

$$
\text { If } \models C \text { holds, then } C \text { is redundant w.r.t. } N \text {. }
$$

Proof: Clearly, any ground instance of $C$ is a ground tautology and redundant, since it follows from the empty set which "contains" only smaller clauses. Qed.

Furthermore, we also note that removing $C$ has no effect on any run of the calculus, since no instance of $C$ can be ever a conflict clause or imply an assignment.

Proposition 6.4 (Strict Subsumption) Let $C, D$ be clauses, $\sigma$ a substitution, and $N$ a set of clauses.

$$
\text { If } C \sigma \subset D \text {, then } D \text { is redundant w.r.t. } N \cup\{C\} \text {. }
$$

Proof: Let $D \delta$ be a ground instance of $D$. Then $C \sigma \delta \subset D \delta$ and $C \sigma \delta<_{\Gamma} D \delta$ holds, for any induced ordering $<_{\Gamma}$. The latter holds, because $\operatorname{def}(C \sigma \delta)<_{p}$ $\operatorname{def}(D \delta)$ holds in the abstract ordering. 
Thus, $D \delta$ is redundant w.r.t. $\{C \sigma \delta\}$, and so is $D$ w.r.t. $\mathrm{N} \cup\{C\}$, and strict subsumption is admissible. Qed.

Similarly to tautology, removing a subsumed clause has little effect on the calculus, since whenever the subsumed clause is a conflict or a reason clause, the subsuming clause is either a conflict clause or implying the same assignment as well.

Proposition 6.5 (Subsumption Resolution) Let $C, D$ clauses, L a literal, $\sigma$ a substitution, and $N$ a clause set.

$$
\text { If } C \sigma \subseteq D \text { holds, then } D \vee \neg L \sigma \text { is redundant w.r.t. } N \cup\{C \vee L, D\} \text {. }
$$

Proof: Redundancy clearly holds as $D$ subsumes $D \vee \neg L \sigma$. Furthermore, we note that exchanging $D \vee \neg L \sigma$ with $D$ in the presence of $C \vee L$ is a sound step. Thus, subsumption resolution as a rule for reducing a clause is admissible. Qed.

\section{Termination and Completeness}

Just as most related calculi, NRCL is a decision procedure for BS as well, under the regularity conditions of Definition [5.2. Below, we show that regular runs never get stuck and eventually terminate.

Proposition 7.1 A regular run is never stuck, i.e. it terminates with the terminal rules, or one of the other rules is applicable.

Proof: It is enough to show that, unless we already terminated, a rule is always applicable. First, we show that conflict search cannot get stuck.

If $\perp$ is already in one of the clause sets, Failure is applicable and we terminate. Thus, w.l.o.g. assume $\perp \notin \mathrm{N} \cup \mathrm{U}$.

Assume $\Gamma$ is total, i.e. defines each ground atom. Then $I_{\Gamma}$ defines all ground atom occurring in gnd $(\mathrm{N})$, and it either satisfies $\mathrm{N}$ or there is a false ground clause from gnd $(\mathrm{N} \cup \mathrm{U})$. In the first case, Success is applicable, and Conflict in the second case.

If $\Gamma$ is not total, and some undefined ground literal is implied by some ground clause, Propagate is applicable. Otherwise, if no ground literal is implied and there is an undefined ground atom, we can always apply Decide. We note that decisions which define only a single ground atom are never blocked.

Second, assume we are resolving a conflict, i.e. the state indicator is $(C ; \sigma ; \pi)$ for some $C, \sigma$, and $\pi$. If the top literal in $\Gamma$ is a decision and if $(C \sigma ; \pi)$ is assertive, then Backjump is applicable. If it is not assertive, then either Factorize, or case (3) of Backjump is applicable.

If the top literal is a deduced literal, and neither does $C=\perp$ hold, nor is $(C \sigma ; \pi)$ assertive - in these cases Backjump is applicable -, then we check the conditions of Skip. If Skip is not applicable, it satisfies the conditions of Resolve. Therefore, either Skip, Factorize, Resolve must be applicable in this case. Qed.

We show termination through a series of lemmas. First, we prove that both conflict search and conflict resolution always terminate:

Lemma 7.2 Assume $N, \Sigma$ and $\mathcal{D}$ are all finite. Then, a conflict search phase of a regular run always terminates, i.e. leads either to a conflict or to termination. 
Proof: By the finiteness of $\Sigma$, we know that $\mathcal{A}_{\Sigma}$ is also finite. Since a regular run is a series of sound steps, we also know that each application of Propagate and Decide defines at least one formerly undefined ground atom.

Thus, a regular run eventually exhausts these rules, and, since it cannot get stuck by Proposition 7.1, one of the rules Failure, Success, or Conflict has to be applied. And thereby, the conflict search phase in question ends. Qed.

Lemma 7.3 Assume $N, \Sigma$ and $\mathcal{D}$ are all finite. Then, a conflict resolution phase of a regular run always terminates, i.e. leads to the application of Backjump in finitely many steps.

Proof: Let us assign to each intermediate state $(\Gamma ; \mathrm{N} ; \mathrm{U} ; k ;(C ; \sigma ; \pi))$ in a conflict resolution the tuple $(\#(\Gamma) ; \operatorname{gnd}(C \sigma ; \pi))$ as a measure, where $\#(\Gamma)$ denotes the number of elements in $\Gamma$.

Let us order these tuples with the lexicographical ordering $<_{\text {lex }}$ based on the canonical ordering over non-negative integers and $<_{0}$ where $<_{0}$ denotes both the ordering induced by the trail after finding the conflict, and its multiset extension. This ordering is well-founded.

We note that conflict resolution cannot get stuck, see Proposition 7.1. Therefore, it is enough to show that each application of the rules Skip, Resolve, and Factorize strictly decreases our measure.

Skip strictly decreases the size of $\Gamma$, and therefore our measure as well. In the case of Resolve and Factorize, it is enough to give a function satisfying the conditions of Proposition 2.26 between the false instances on the two sides, i.e. a function $\gamma$ which assigns ground clauses from the right-hand side conflict-set to larger ground clauses from the left-hand conflict-set.

First, assume we apply Resolve to the state

$$
\left(\Gamma,\left(L^{\prime} \cdot \sigma^{\prime} ; \pi^{\prime}\right)^{C^{\prime} \vee L^{\prime}} ; \mathrm{N} ; \mathrm{U} ; k ;(C \vee L ; \sigma ; \pi)\right)
$$

and we get

$$
\left(\Gamma,\left(L^{\prime} \cdot \sigma^{\prime} ; \pi^{\prime}\right)^{C^{\prime} \vee L^{\prime}} ; \mathrm{N} ; \mathrm{U} ; k ;\left(\left(C \vee C^{\prime}\right) \eta_{0} ; \sigma^{*} ; \pi \eta, \pi^{\prime} \eta\right)\right)
$$

where $\eta=\operatorname{mgu}\left(L^{\prime} \sigma^{\prime}, \neg L \sigma\right), \eta_{0}=\operatorname{mgu}\left(L^{\prime}, \neg L\right)$, and $\sigma^{*}$ such that $\eta_{0} \sigma^{*}=\sigma \sigma^{\prime} \eta$. For the sake of readability, let us introduce the symbols $\alpha=\sigma \sigma^{\prime} \eta$ and $\pi^{*}=$ $\pi \eta, \pi^{\prime} \eta$.

Now, let $\beta$ be a grounding substitution such that $\left(C \vee C^{\prime}\right) \alpha \beta \in \operatorname{gnd}((C \vee$ $\left.\left.C^{\prime}\right) \alpha ; \pi^{*}\right)$. Since it was derived via resolution, there is a corresponding valid ground resolution step with premises

- $C_{1} \vee L_{1} \in \operatorname{gnd}((C \vee L) \alpha ; \pi \eta)$

- $C_{2} \vee L_{2} \in \operatorname{gnd}\left(\left(C^{\prime} \vee L^{\prime}\right) \alpha ; \pi^{\prime} \eta\right)$

where we assume $L_{1}$ and $L_{2}$ are the literals corresponding to $L$ and $L^{\prime}$, respectively. Since we apply resolution, we also know that $L_{1}=\neg L_{2}$, and $\left(C \vee C^{\prime}\right) \alpha \beta=C_{1} \vee C_{2}$.

By the definition of sound states and Propagate, we know that $C_{2}$ contains only literals which were defined before the last assignment, and thus, $C_{2}<_{0}$ (ᄀ) $L_{2}$, and therefore $C_{2}<_{0} L_{1}$. Then, $C_{1} \vee C_{2}<_{0} C_{1} \vee L_{1}$ must hold, and thus, we shall define $\gamma\left(C_{1} \vee C_{2}\right)$ as $C_{1} \vee L_{1}$. 
Since $\gamma$ can be defined over the whole $\operatorname{gnd}\left(\left(C \vee C^{\prime}\right) \alpha ; \pi^{*}\right)$ and $\operatorname{gnd}((C \vee$ $L) \alpha ; \pi \eta)$ is a subset of $\operatorname{gnd}((C \vee L) \sigma ; \pi)$, we can apply Proposition 2.26, and we get

$$
\operatorname{gnd}((C \vee L) \sigma ; \pi)>_{0} \operatorname{gnd}\left(\left(C \vee C^{\prime}\right) \alpha ; \pi^{*}\right)
$$

and our measure strictly decreases, as the size of the trail is unchanged. The proof for Factorize is analogous. Qed.

Next, we show that only finitely many new clauses can be learned thanks to our non-redundancy results in Theorem 6.2

Lemma 7.4 If $N, \Sigma$ and $\mathcal{D}$ are finite, a regular run can only learn finitely many new clauses.

Proof: We use Higman's Lemma [15 to prove this claim. The lemma states that given an infinite sequence $w_{1}, w_{2}, \ldots$ of words over a finite alphabet, there is always an index $i$ and a subsequent index $j$ such that the word $w_{i}$ is embedded into $w_{j}$, i.e. after deleting some letters from $w_{j}$ we can get $w_{i}$.

Now, consider $\mathcal{A}_{\Sigma}$. Since $\Sigma$ and $\mathcal{D}$ are finite, both the set of ground atoms and ground literals over $\Sigma$ and $\mathcal{D}$ are finite. The latter serves as the finite alphabet for our proof.

Since every learned clause is non-redundant at the time they are learned, by Theorem 6.2, we can assign a non-redundant ground instance to any learned clause, by the definition of redundancy.

Assume we learn infinitely many clauses, and let us consider the assigned ground clauses $C_{1}, C_{2}, \ldots$, where $C_{1}$ is assigned to the clause learned at the first conflict, $C_{2}$ to the clause learned at the second, and so on.

Now, take any term ordering $>$, order the literals of the clauses, and assign this ordered sequence of literals to each clause. Let us denote this word over the alphabet of ground literals by $w(C)$ for every ground clause $C$.

Then, by Higman's Lemma, there are indices $i<j$ such that $w\left(C_{i}\right)$ is embedded in $w\left(C_{j}\right)$. But it means that $C_{i} \subseteq C_{j}$, i.e. $C_{j}$ is strictly subsumed by or equal to $C_{i}$.

The admissibility of strict subsumption was proven in Proposition 6.4, and clearly an already present ground clause cannot be non-redundant either, for any induced ordering. Thus, $C_{j}$ cannot be redundant at the $j$ th conflict, a contradiction. Qed.

Finally, we show termination, and state the main result as a corollary.

Theorem 7.5 (Termination) A regular run always terminates if $N, \Sigma$ and $\mathcal{D}$ are finite.

Proof: First, we note that a run can be seen as a series of conflict search and conflict resolution phases, which ideally ends with a terminal rule. By Lemma 7.2, Lemma 7.3, and Proposition 7.1, we know that each phase ends after finitely many steps without getting stuck.

Thus, an infinite run must be an infinite series of conflict search and resolution sequences. Since each conflict resolution ends with Backjump, it would imply that infinitely many new clauses are learned. But it contradicts Lemma 7.4 Qed. 
Corollary 7.6 (Decision Procedure) Regular runs provide a decision procedure for the Bernays-Schönfinkel fragment if $N, \Sigma$ and $\mathcal{D}$ are finite.

I.e. every regular run terminates after finitely many steps with Failure, or Success, for an unsatisfiable, or satisfiable clause set $N$, respectively.

Proof: It follows from Proposition 7.1 and the Theorems 4.4 and 7.5. Qed.

\section{Towards Implementation}

This far we considered mostly our calculus in an abstract fashion, and it is enough to establish the results of the previous chapters.

Here, we elaborate some details regarding the constraints, and refine some steps to bring NRCL closer to practical application. In particular, we provide an abstract algorithm for exhaustive propagation, to highlight some important difficulties and expensive steps in the calculus.

However, this section does not aim to provide a complete abstract algorithm for regular runs, we only briefly address some challenges and propose some solutions and approaches, which provides us a starting point for later implementation and experimentation.

\subsection{Free Variables}

The definition of normal form for constrained literals demands the left-hand side of a constraint to contain only variables occurring in the constrained literal. Our calculus derives new assignments, i.e. new constrained literals for $\Gamma$, by applying resolution between the literals in $\Gamma$ and the clauses in $\mathrm{N} \cup \mathrm{U}$.

However, even after normalization, the resulting candidate $(L \cdot \sigma ; \pi)^{C}$ might contain free left-hand side variables, i.e. variables which occur in the reason clause instance $C \sigma$, and still occur in $\operatorname{lvar}(\pi)$, but do not occur in $L \sigma$. The following example demonstrates this behaviour.

Example 8.1 Let us take

$$
N=\left\{C_{1}: \neg Q(x, x), C_{2}: \neg Q(x, y) \vee \neg Q(x, z) \vee P(y, z)\right\}
$$

And assume that after an application of Propagate and Decide we get the trail

$$
\Gamma=(\neg Q(x, x) ; \top)^{C_{1}},(Q(x, y) ;(x, y) \neq(v, v))^{1}
$$

Now, applying Propagate between $\Gamma$ and the clause $C_{2}$, we get the constrained literal

$$
(P(y, z) ;(x, y) \neq(v, v) \wedge(x, z) \neq(w, w))
$$

Over $\mathcal{D}=\{a, b\}$, this constraint is satisfiable, the cover-set is $\{P(a, a), P(b, b)\}$, and after eliminating the free variable $x$ we get the constrained literals

$$
(P(y, z) ; y \neq a \wedge z \neq a) \text { and }(P(y, z) ; y \neq b \wedge z \neq b)
$$

Semantically, these variables are to be treated as existential variables, of course. These variables cause two problems. 
First, in the presence of these existentially handled variables our constrained literal set for difference defined in Lemma 2.16 is no longer valid. In particular disjointness is no longer guaranteed.

A simple way to overcome this issue is to split the resulting literal into a set of literals by instantiating the free left-hand side variables in every possible way, as seen in the example above. This elimination procedure results in a set of not necessarily disjoint constrained literals.

Second, while eliminating these variables might be a solution, we still need to store the instantiating assignments. This information is used when applying the rules Resolve and Factorize during conflict resolution. This is already accomplished through using closures as introduced in Section 3.

\subsection{Indexing Scheme}

In the propositional setting, the watched literal scheme watches two literals in every non-unit clauses. These literals are assumed to be true or undefined under the current model assumption, or all literals but a single watched literal are false in the clause.

Whenever a new assignment makes a watched literal false, we attempt to find a new non-false literal. If it is not possible, the other watched literal is propagated resulting either in a new assignment or a new conflict clause.

This scheme enables efficient propagation at small computational costs as it cuts back the number of clauses we have to consider after a new assignment and requires no additional bookkeeping during backtrack.

When lifting the scheme, we have to keep in mind that manipulating our constraints is more expensive. Therefore, a direct lifting of the technique by exactly maintaining which literals are watched in the different instances of a clause would be too expensive for our purposes.

Here, we propose a lightweight approach which uses two levels of indexing the literals of the current clause set. Every clause is indexed by one of these levels, but not both.

The first level attempts to mimic the two-watched-literal scheme, and indexes only two literals in the clauses. We can choose the interpretation of watching a literal $L$ as an approximation of cannot be false by selecting one of the following:

- $\nexists\left(L^{\prime} \cdot \sigma ; \pi\right)^{\alpha} \in \Gamma: \exists \operatorname{mgu}\left(\neg L^{\prime} \sigma, L\right)$

- $\nexists\left(L^{\prime} \cdot \sigma ; \pi\right)^{\alpha} \in \Gamma: \exists \delta=\operatorname{mgu}\left(\neg L^{\prime} \sigma, L\right)$ and $\pi \delta \neq \perp$

- $\exists\left(L^{\prime} \cdot \sigma ; \pi\right)^{\alpha} \in \Gamma: \exists \delta=\operatorname{mgu}\left(\neg L^{\prime} \sigma, L\right)$ and $\pi \delta$ is not empty

Obviously, the last choice is the most expensive and the first two should be preferred.

Whenever a new assignment is made, we first try to adjust the watched literals on level one. If a clause contains no longer two appropriate literals, we push it to the second level. On this level we index all literals of the clauses, e.g. in a context tree with top-level symbol hashing.

Putting clauses back to level one can be done either by maintaining an activity heuristics and time to time manually check for watchable literals, or managing lists of pointers for all clause-literals to relevant assignments on the trail. 
This topology should make propagation cheaper, and in particular using level one should make it easier to ignore clauses irrelevant w.r.t. the recent assignments.

\subsection{Finding Candidates}

Before we propose an abstract algorithm for exhaustive propagation, we introduce a simple derivation system for finding candidates. Of course, in the actual implementation this system will be replaced by more efficient algorithms on the indexing structures.

The rules work on tuples of the form $(C ; \sigma ; \pi)^{i}$ where

- $C$ is a clause, a subclause of some initial clause $C_{0}$ from the current clause set

- $\sigma$ is a substitution over $\operatorname{var}\left(C_{0}\right)$

- $\pi$ is a dismatching constraint

- $i$ is the number of application of the last assignment of the trail, which has relevance in the next section

The initial tuple for a clause $C_{0} \in \mathrm{N} \cup \mathrm{U}$ is $\left(C_{0} ; \emptyset ; \top\right)^{0}$ and we try to resolve each literal in $C$ with the following rule:

$$
(C \vee L ; \sigma ; \pi)^{i} \Rightarrow_{\Gamma}\left(C ; \sigma \theta ; \pi \theta \wedge \pi^{\prime} \theta\right)^{i^{\prime}}
$$

Where there is a $\left(\neg L^{\prime} \cdot \sigma^{\prime} ; \pi^{\prime}\right)^{\alpha} \in \Gamma$ such that

- $\exists \theta=\operatorname{mgu}\left(L \sigma, L^{\prime} \sigma^{\prime}\right)$

- $\left(\pi \theta \wedge \pi^{\prime} \theta\right) \neq \perp$ and normalized

- $i^{\prime}$ is $i+1$ if $\left(\neg L^{\prime} \cdot \sigma^{\prime} ; \pi^{\prime}\right)^{\alpha}$ is the last assignment in $\Gamma$, and $i$ otherwise

Applying this rule we can get candidates for the rules Conflict, and Propagate by deriving respectively tuples of the form

- $(\perp ; \sigma ; \pi)^{i}$, or

- $(L ; \sigma ; \pi)^{i}$

We note that non-emptiness is not checked fully, only a cheaper precondition of it. Free left-hand side variables and already defined instances are not removed either.

\subsection{Exhaustive Propagation}

In this section, we propose the abstract algorithm PROP for exhaustive propagation with conflict detection. It basically processes a queue $\mathrm{PQ}$ of candidates for new assignments. As an invariant, we assume each constrained literal in the queue

1. has a normalized non- $\perp$ constraint

2. consistent with the current $\Gamma$

3. contains no free left-hand side variable 


\section{PROP}

Initially, this queue consists of the literals induced by the unit clauses. Unit clauses has to be checked for contradiction prior calling PROP. When calling after decisions, $\mathrm{PQ}$ is assumed to contain the immediate consequences of the decision. Checking for blocking should generate this set anyway.

PROP processes the literals on PQ. First, it removes already defined instances by calling the function DIFF. This produces a set of disjoint and undefined constrained literals, each of which is a valid subject of Propagate. See Section 2.3 for the definition of the difference operation " -", and see below the abstract algorithm for DIFF.

These literals are then checked for emptiness, added to $\Gamma$ and set to true. Their consequences - conflicts and new candidates for PQ - are then generated by addConsequences.

We continue this process until PQ gets empty, or a conflict is found. The first indicates the finished exhaustive application of Propagate, and Decide can be called. In this case we return true. And in the latter case, we return false, and the found conflict is stored in conflictSet.

On the course of this section, we might use the symbol $\ell$ to denote annotated constrained literals, and the following auxiliary functions:

- pop: removes an element of a queue, list, or set

- notEmpty: carries out a full non-emptiness check for a constraint or constrained literal

- addAssignment: adds a new assignment to $\Gamma$ (and its indexing structures)

- $\operatorname{cunIF}(\ell, \Gamma)$ : Finds the literals in $\Gamma$ which are unifiable with $\ell$, and returns an array of them and its size

- NF: normalizes a constraint, constrained literal, or a set of constrained literals, as described in Subsection 2.2. In the latter case, it removes resulting literals with $\perp$-constraints.

- freeLVars: produces the set of free left-hand side variables of a constrained literal

- selectOne: randomly, or heuristically selects an element of a set, or a list

- adjustLevel1: adjusts the first index level for clauses after a new assignment given as parameter, as described in Section 6.2.

- getCandidates: provides the list of indexed clauses which contains a literal unifiable with the complement of a given literal

\section{DIFF}

It iteratively removes the already defined instances from the proposed assignment. The result is a set of disjoint and undefined constrained literals with non- $\perp$ constraints. 

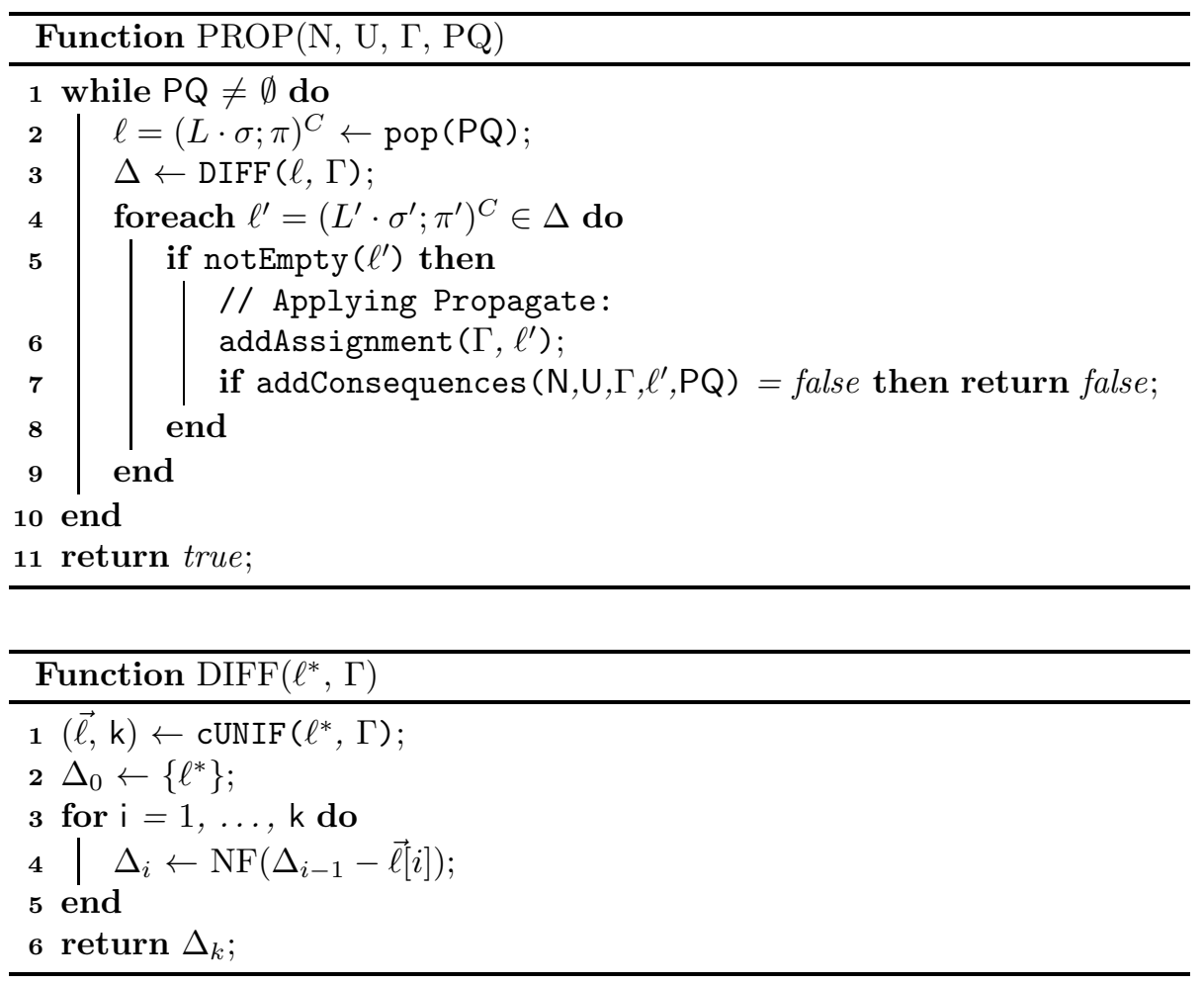

\section{elimFV}

An auxiliary function for finding new candidates. It iteratively removes the free left-hand side variables, and only keeps the literals with non- $\perp$ normalized constraints.

\section{addConsequences}

Finally, addConsequences checks whether a new assignment produces a conflict and generates new candidates for PQ. It returns true if no conflict is found, and false otherwise. If a conflict is found, it is saved in conflictSet.

We distinguish two types of conflicts. It is easy to see, that if the new assignment is used only once in deriving a conflict, then PQ must already hold an unprocessed candidate which is falsified by the new assignment. Thus, we check PQ first for a contradiction, and start generating new candidates with $\Rightarrow_{\Gamma}$ only afterwards.

We then use the derivation system of 6.3 to derive new constrained literals. We only consider derivations where the latest assignment has to be used at least once. If it is used only once we can be sure the new literal is not false. If it is not the case, we check for a possible conflict.

As stated before, in the actual implementation the proper retrieval algorithms will eliminate the inefficiency of considering all derivations.

Finally, the new candidates are tested for free variables, and they are removed if there are any. 


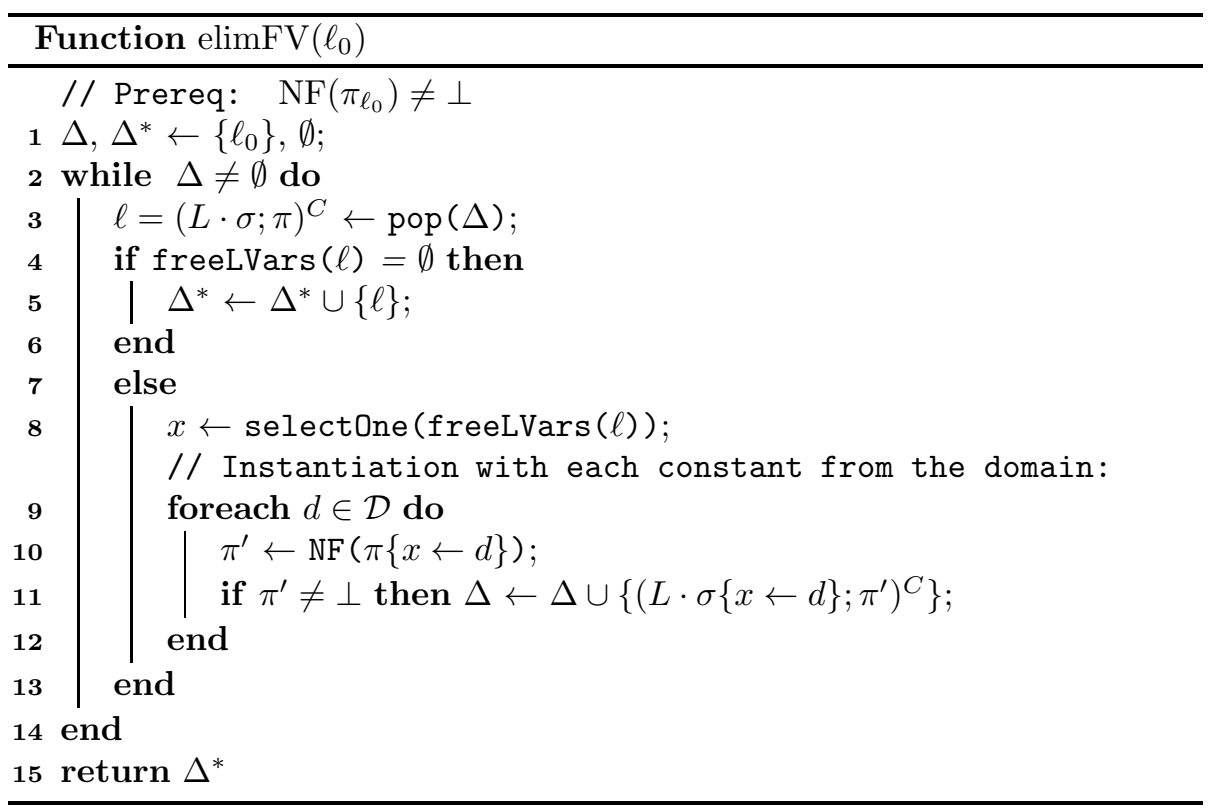

\subsection{Picking the Next Decision}

When making a new decision, we a pick a candidate $(L ; \pi)$, remove all the already defined instances, and then test all immediate conflicts for blocking.

If there is a blocking conflict, we might then either pick an entirely new decision candidate, or try to fix $(L ; \pi)$ by instantiating some variables in $L$, and thereby generating a new set of candidates.

This can be achieved by picking a blocking ground instance $C^{\prime}=C \delta$ which contains $\neg L \delta_{1}, \neg L \delta_{2}$ such that both $L \delta_{1}, L \delta_{2} \in \operatorname{gnd}(L ; \pi)$ and $L \delta_{1} \neq L \delta_{2}$ holds. Now, choose a variable for which $x \delta_{1} \neq x \delta_{2}$, and split $(L ; \pi)$ into $(L\{x \leftarrow$ $\left.\left.x \delta_{1}\right\} ; \pi\left\{x \leftarrow x \delta_{1}\right\}\right)$ and $\left(L ; \pi \wedge x \neq x \delta_{1}\right)$. By instantiating further variables, we eventually get a decision which is not blocking, since a ground decision is always suitable.

A non-blocking decision is then added to $\Gamma$, and whether we found a nonblocking conflict or not, we continue with conflict resolution or with calling PROP after generating the immediate propagation candidates in a similar way as in addConsequences.

Initially, the set of decision candidates are generated from the literals occurring in N. This set can be later refined by the above steps, and individual candidates might be substituted with sets of new candidates.

Since removing defined instances is always relative to the current $\Gamma$, it has to be guaranteed that the set of all possible candidates covers the original set. It can be ensured for example by keeping a trail for these refinement steps as well, and re-roll them in parallel with the backtracking procedure.

\subsection{Ranking Literals}

Most current SAT solvers also employ variable selection schemes based on dynamic ranking of propositional variables. This technique rewards variables in- 


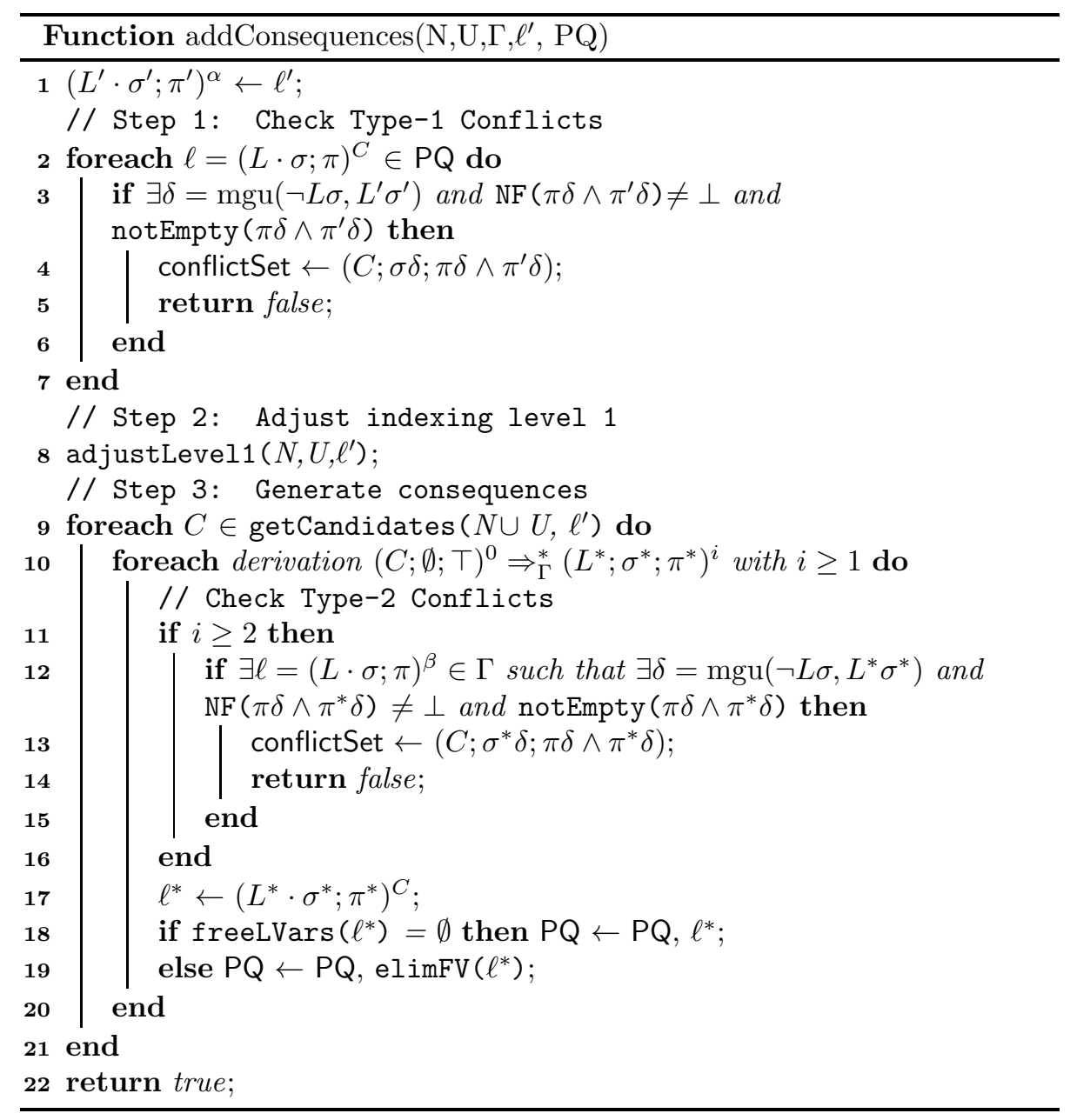


volved in recent conflicts, and proved itself efficient in the propositional context.

Following the footsteps of the now classic decaying variable sum, we reward the literals involved in the clause learning phase following the latest conflict.

This is accomplished by maintaining a list of literals and scores. Whenever some literal $L$ is added to the clause of the intermediate state, we add a pair $(L ; v)$ to this list.

To focus on recent conflicts, we increase $v$ gradually, and occasionally we reset $v$ to some initial value and normalize the list. The latter can be triggered upon reaching some extreme value, automatically after a certain number conflicts, or at restarts.

Restarts are commonly used in SAT solvers to redirect the focus of the search using the learned clauses and the current variable scores. Applying it only finitely many times does not violate completeness.

Then, whenever we need to choose a new decision, we rank the candidates by combining the scores belonging to literals which are unifiable with the candidate in question. As an example we propose addition or maximum. We then choose the literal with the highest combined score.

\subsection{Clause Learning and Backjumping}

As the conflicts are now discovered, every conflict-set uniquely assigns a $\Gamma$ assignment to each literal of the conflict clause. This make detecting assertiveness easy and spares us a number of emptiness checks, as they are already done during conflict detection. This way, the only non-deterministic choice is the application of Factorize versus Resolve, when both is applicable.

Once a new clause is learned, a suitable backtrack level is needed. Should we learn only the ground clauses in $\operatorname{gnd}(C \sigma ; \pi)$ when the last conflict-set is $(C ; \sigma ; \pi)$, we could determine the backtrack position at ease, similarly to the propositional solvers.

But we learn the more general $C$, and the right backtrack position has to be computed from all the instances of $C$. We have to consider all conflicting instance of $C$ w.r.t. $\Gamma$, and for each instance, we have to determine a minimal backtrack position. Then, we backjump to the minimum of these positions.

Without providing more details, we only note that some instances produce new assignments after backtrack, some might block existing decisions, and some might even be new conflicts after backjump.

\section{Related Work}

In this section, we briefly compare NRCL to existing solutions. As BernaysSchönfinkel problems can be successfully handled with finite model finders as well, we cover both BS-specific techniques and more general finite model building approaches. In the case of the latter systems, we focus on their behavior on the Bernays-Schönfinkel fragment.

The first successful approaches to finite model building were Mace and SEM, see e.g. 31. The early version of Mace flattens and grounds the given clause set, and passes it on to a SAT solver. This approach is developed further by Paradox [10. 
Compared to this approach, we work directly with the first-order clause set instead of the often exponentially larger set of ground instances.

The latest version of Mace [22] follows the approach of SEM [33] and FINDER [30. Instead of generating the ground instances, it maintains the function and predicate tables, and fills them out using a sophisticated backtracking algorithm.

Compared to this approach, we represent the model implicitly via constrained literals, and let the learned clauses guide our calculus.

Over the last decade several attempts were made to lift the DPLL algorithm. Model Evolution [7] and its implementation Darwin [5] represents a model with a set of first-order literals, called context, and utilizes a backtracking algorithm which detects conflicts via a propositional approximation. The latter approach requires numerous extra branchings in Darwin.

It is refutationally complete over first-order clauses and provides a decision procedure for the Bernays-Schönfinkel fragment. Its extension [6] enriches the calculus with the ability to learn lemmas at conflicts.

Compared to Model Evolution, NRCL does not need expensive approximations to find conflicts, we treat redundancy, and we consider our model representation easier to maintain. We also note that Model Evolution is a lifting of DPLL, and relies on splitting the clauses and backtracking, while our search makes progress with backjumping guided by clauses learned with resolution.

Furthermore, we handle redundancy and show that the clauses learned by our calculus are non-redundant. Finally, it was shown in [12 that using contexts might result in exponentially larger model representations.

We note that this result holds for the general case with function symbols, but in our setting e.g. the constrained literal

$$
\left(P\left(x_{1}, x_{2}, \ldots, x_{k}\right) ; x_{1} \neq x_{2} \wedge x_{2} \neq x_{3} \wedge \ldots x_{k-1} \neq x_{k}\right)
$$

whose size is $O(k)$, requires a representation of size at least $O\left(k^{2}\right)$ as a context. Thus, at least a quadratic relation holds even for the Bernays-Schönfinkel fragment.

$D P L L(S X)$ 27] attempts to lift DPLL to BS in the same manner as we do, and uses substitution sets represented by BDDs as constraints. Substitution sets provide an explicit way to represent models.

It is well-known that in the general setting with function symbols implicit representations have stronger expressive power 26] 20. In our setting, explicit representations have the potential to be exponentially larger then the corresponding implicit representations.

The following simple example demonstrates this claim. Over $\mathcal{D}=\{a, b, c\}$, consider the constrained literal

$$
\left(P\left(x_{1}, \ldots, x_{k}\right) ; x_{1} \neq x_{2} \wedge x_{2} \neq x_{3} \wedge \cdots \wedge x_{k-1} \neq x_{k}\right)
$$

Then it is easy to see that the corresponding explicit representation is made up of all the ground instances covered by this literal.

Therefore, while the size of the implicit representation increases linearly in $k$, the size of the corresponding explicit representation is $O\left(2^{k}\right)$, i.e. increases exponentially in $k$.

The authors of this paper are convinced that this exponential blow-up happens whenever in the implicit representation has no finite explicit representation 
(see 26 20 for details) in the language enriched with a function symbol. However, this conjuncture needs further consideration, and we leave it for future work.

Furthermore, compared to $\operatorname{DPLL}(S X)$ our approach is more modular as it allows the use of arbitrary constraint language, restricted only by the operations we expect. We also address redundancy, and exploit the non-redundancy result to show termination, which we consider a valuable addition.

The most recent calculus SGGS, introduced in [9], promises a semantically guided, goal sensitive, model-based proof system. It uses simple constraints, socalled standard forms, conjunctions of negative atomic constraints of the form $x \neq y$, or $\operatorname{top}(x) \neq f$.

Then, a model is represented by a sequence of constrained clauses with selected literals. This sequence overrides a given initial interpretation $I$, which serves both as initial model assumption and as semantic guidance for the calculus.

The procedure then keeps expanding this sequence in order to satisfy more and more clauses, and handles contradictions via resolution and splitting the constrained clauses to maintain an invariant - every literal in every clause in the sequence must have either only false, or only true instances w.r.t. I and the constraints.

NRCL utilizes a more expressive constrained language, which allows tuples to be used. This results in less fragmentation of the representation, i.e. SGGS might need several constraints in standard form to express a single dismatching constraint of our calculus.

This allows us to learn more general clauses, and also potentially decreases the size of the representation. Our model representation relies on constrained literals instead of clauses, and we consider it to be more explicit than the approach of SGGS which requires identifying the constrained instances of the clauses which are indeed producing new assignments.

Finally, the resolution applied by SGGS only repairs the model, it can be discarded later as the search progresses, and the splittings applied to maintain the invariants also forces the result of resolution to be more specific, more local. Compared to this, our calculus learns and saves new clauses, uses backjumping, and we proved these clauses are non-redundant.

We also mention geometric resolution 24 which uses a special normal form called geometric normal form. In this calculus the formulas themselves constitute the rules of a system based on backtracking. Through the inference geometric resolution it also provides a way to learn new formulas. The transformation to geometric normal form also includes flattening, which our approach avoids.

The calculus Inst-Gen [14 and its implementation iProver 17 has been quite successful at solving Bernays-Schönfinkel problems, and competitive even for the first-order fragment. It generates a propositional approximation of the clause set by instantiating all the variables with constants, and passes it on to a SAT solver.

Unsatisfiability of the approximation entails the unsatisfiability of the original problem. On the other hand, if an abstract model is generated, it is used to guide the calculus to add proper instances of the original clauses, which refines the propositional abstraction.

This procedure is continued then, until either unsatisfiability is proven, or 
saturation is achieved, which implies that the abstract model can be lifted to a first-order model for the original clause set.

The algorithm is further enhanced by using dismatching constraints, and applying redundancy elimination based on generating first-order resolvents for subsumption with a theorem prover, and finding simplification candidates efficiently with ground reasoning.

Compared to iProver, our approach is fine-grained, as the evaluation and refinement of our abstraction happen interleaved with the other reasoning steps. Furthermore, we work directly with the original clause set, and our trail always corresponds to a consistent first-order model candidate.

Finally, even general purpose first-order theorem provers implement specialized techniques to handle Bernays-Schönfinkel problems.

Generalisation introduced in 25] for Vampire is an additional technique for resolution-based saturation. It infers $P(x)$ if $P(c)$ has been established for all relevant constant $c$. Coupled with efficient sort inference, it has the potential to exponentially speed up theorem proving.

The technique introduced in [16] for SPASS employs a combination of restricted superposition on Horn clauses, and labelled splitting [13] on non-Horn clauses.

Compared to these approaches, NRCL maintains a model candidate, it is restricted to learn clauses only at conflicts and only non-redundant ones, contains no Horn clause restrictions, and the implicit branchings through decisions and backjumps are more elaborate and guided by the model search, compared to the splitting techniques employed by first-order theorem provers.

\section{Conclusion}

In this paper, we proposed the decision procedure NRCL for the BernaysSchönfinkel fragment. Our approach represents a model candidate as a set of constrained literals, and establishes a model or a proof of unsatisfiability through a series of decisions, propagations, and learning new clauses.

Our work closely relates to $D P L L(S X)$ [27, which introduces a similar calculus, and the more recent calculus $S G G S[9]$. Compared to earlier work in this direction, we investigated the standard redundancy notion w.r.t. the ordering induced by the current trail. In particular, we proved that each learned clause is non-redundant in our calculus, and we based our termination proof on this result.

In Section 8, we addressed some of the difficulties of this approach, and provided details for implementation. Finally, we gave a brief comparison to the existing solutions in Section 9.

As future research, the immediate goal is to make an efficient implementation of NRCL. This includes developing suitable and efficient term indexing structures, possibly revising the constraint language, and defining concrete and efficient heuristics for selecting decisions.

On the other hand, the long-term goal of our research is to extend this calculus beyond Bernays-Schönfinkel. The next step into this direction is to enrich our calculus with function symbols and sorts to handle the non-cyclic fragment introduced in [19. This fragment still has the finite Herbrand model 
property, thus, we are reasonably convinced that our results will directly extend to this fragment.

The further goals are to consider fragments decidable with superposition, to introduce equality into our calculus, and finally to extend our work to finite model finding.

\section{References}

[1] A. Armando, P. Baumgartner, and G. Dowek, eds. Automated Reasoning, 4th International Joint Conference, IJCAR 2008, Sydney, Australia, August 12-15, 2008, Proceedings, 2008, LNCS 5195. Springer.

[2] L. Bachmair and H. Ganzinger. Resolution Theorem Proving. In Robinson and Voronkov [28, pp. 19-99.

[3] L. Bachmair, H. Ganzinger, C. Lynch, and W. Snyder. Basic Paramodulation and Superposition. In D. Kapur, ed., Automated Deduction - CADE11, 11th International Conference on Automated Deduction, Saratoga Springs, NY, USA, June 15-18, 1992, Proceedings, 1992, LNCS 607, pp. 462-476. Springer.

[4] L. Bachmair, H. Ganzinger, C. Lynch, and W. Snyder. Basic Paramodulation. Inf. Comput., 121(2):172-192, 1995.

[5] P. Baumgartner, A. Fuchs, U. Koblenz-landau, F. Informatik, C. Tinelli, and B. F. Tinelli. Darwin: A Theorem Prover for the Model Evolution Calculus, 2004.

[6] P. Baumgartner, A. Fuchs, and C. Tinelli. Lemma Learning in the Model Evolution Calculus. In M. Hermann and A. Voronkov, eds., LPAR, 2006, LNCS 4246, pp. 572-586. Springer.

[7] P. Baumgartner and C. Tinelli. The Model Evolution Calculus. In F. Baader, ed., CADE, 2003, LNCS 2741, pp. 350-364. Springer.

[8] A. Biere, M. Heule, H. van Maaren, and T. Walsh, eds. Handbook of Satisfiability, 2009, Frontiers in Artificial Intelligence and Applications, vol. 185. IOS Press.

[9] M. P. Bonacina and D. A. Plaisted. SGGS theorem proving: an exposition. Notes of the Fourth Workshop on Practical Aspects in Automated Reasoning (PAAR), Seventh International Joint Conference on Automated Reasoning (IJCAR) and Sixth Federated Logic Conference (FLoC), Vienna, Austria, July 2014., 2014.

[10] K. Claessen and N. Srensson. New Techniques that Improve MACE-style Finite Model Finding. In Proceedings of the CADE-19 Workshop: Model Computation - Principles, Algorithms, Applications, 2003.

[11] H. Comon. Disunification: A Survey. In Computational Logic - Essays in Honor of Alan Robinson, 1991, pp. 322-359. 
[12] C. G. Fermüller and R. Pichler. Model Representation via Contexts and Implicit Generalizations. In R. Nieuwenhuis, ed., CADE, 2005, LNCS 3632, pp. 409-423. Springer.

[13] A. Fietzke and C. Weidenbach. Labelled splitting. In Ann. Math. Artif. Intell. Vol. 55 No. 1-2, 2009, pp. 3-34.

[14] H. Ganzinger and K. Korovin. New Directions in Instantiation-Based Theorem Proving. In LICS, 2003, pp. 55-64. IEEE Computer Society.

[15] G. Higman. Ordering by Divisibility in Abstract Algebras. Proceedings of the London Mathematical Society, s3-2(1):326-336, 1952.

[16] T. Hillenbrand and C. Weidenbach. Superposition for Bounded Domains. In M. P. Bonacina and M. Stickel, eds., McCune Festschrift, 2013, LNCS 7788, pp. 68-100. Springer. Based on the Research Report MPI-I-2007RG1-002.

[17] K. Korovin. iProver - An Instantiation-Based Theorem Prover for FirstOrder Logic (System Description). In Armando et al. [1], pp. 292-298.

[18] K. Korovin. Inst-Gen - A Modular Approach to Instantiation-Based Automated Reasoning. In A. Voronkov and C. Weidenbach, eds., Programming Logics, 2013, LNCS 7797, pp. 239-270. Springer.

[19] K. Korovin. Non-cyclic Sorts for First-Order Satisfiability. In P. Fontaine, C. Ringeissen, and R. A. Schmidt, eds., FroCos, 2013, LNCS 8152, pp. 214-228. Springer.

[20] J.-L. Lassez and K. Marriott. Explicit Representation of Terms Defined by Counter Examples. J. Autom. Reasoning, 3(3):301-317, 1987.

[21] H. R. Lewis. Complexity Results for Classes of Quantificational Formulas. J. Comput. Syst. Sci., 21(3):317-353, 1980.

[22] W. McCune. Mace4 Reference Manual and Guide. CoRR, cs.SC/0310055, 2003.

[23] R. Nieuwenhuis and A. Rubio. Paramodulation-Based Theorem Proving. In Robinson and Voronkov [28, pp. 371-443.

[24] H. de Nivelle and J. Meng. Geometric Resolution: A Proof Procedure Based on Finite Model Search. In U. Furbach and N. Shankar, eds., IJCAR, 2006, LNCS 4130, pp. 303-317. Springer.

[25] J. A. N. Pérez and A. Voronkov. Proof Systems for Effectively Propositional Logic. In Armando et al. 1], pp. 426-440.

[26] R. Pichler. Explicit versus implicit representations of subsets of the Herbrand universe. Theor. Comput. Sci., 290(1):1021-1056, 2003.

[27] R. Piskac, L. M. de Moura, and N. Bjørner. Deciding Effectively Propositional Logic Using DPLL and Substitution Sets. J. Autom. Reasoning, 44(4):401-424, 2010. 
[28] J. A. Robinson and A. Voronkov, eds. Handbook of Automated Reasoning (in 2 volumes). Elsevier and MIT Press, 2001.

[29] J. P. M. Silva and K. A. Sakallah. Conflict Analysis in Search Algorithms for Satisfiability. In ICTAI, 1996, pp. 467-469.

[30] J. K. Slaney. FINDER: Finite Domain Enumerator - System Description. In A. Bundy, ed., CADE, 1994, LNCS 814, pp. 798-801. Springer.

[31] T. Tammet. Finite Model Building: Improvements and Comparisons. In In: Model Computation Principles, Algorithms, Applications, CADE-19 Workshop W4, 2003.

[32] C. Weidenbach. Combining Superposition, Sorts and Splitting. In Robinson and Voronkov 28, pp. 1965-2013.

[33] J. Zhang and H. Zhang. SEM: a System for Enumerating Models. In IJCAI, 1995, pp. 298-303. Morgan Kaufmann. 